\title{
Factores que explican el apoyo social del adulto mayor en tres ciudades de Colombia. 2016
}

\section{Factors that explain the social support of the elderly in three cities of Colombia. 2016}

\author{
Douglas Lizcano Cardona 1 ORCID, Doris Cardona Arango 2 ORCID Angela Segura Cardona \\ Alejandra Segura Cardona 40 RCID , Maite Catalina Agudelo-Cifuentes ${ }^{5} \underline{0 R C I D}$, Diana Muñoz \\ Rodríguez $z^{6} \mathrm{ORCID}$ \\ Universidad CES \\ Colombia
}

Fecha correspondencia:

Recibido: marzo 24 de 2019.

Aceptado: abril 29 de 2020.

Forma de citar:

Lizcano Cardona, D., Cardona

Arango, D., Segura Cardona, A.,

Segura Cardona, A., Agudelo-

Cifuentes, M.C., \& Muñoz Rodríguez,

D. (2020). Factores que explican el apoyo social del adulto mayor en tres ciudades de Colombia. 2016. Rev. CES Psico, 13(2), 144-165.

\section{Open access \\ (c) Copyright \\ Licencia creative commons \\ Ética de publicaciones \\ Revisión por pares \\ Gestión por Open Journal System \\ DOl: http://dx.doi.org/10.21615/ \\ cesp. 13.2.10 \\ ISSN: 2011-3080}

Sobre los autores:

1. Estudiante de Doctorado en

Epidemiología y Bioestadística.

Magister en Epidemiología.

\section{Resumen}

Objetivo: identificar los factores asociados a las dimensiones del apoyo social del adulto mayor en tres ciudades de Colombia: Medellín, Barranquilla y Pasto. Método: estudio cuantitativo, transversal analítico, de fuente de información primaria, obtenida a partir de encuestas realizadas a adultos mayores sobre aspectos demográficos, familiares y sociales, y escalas de medición validadas que permitieron evaluar aspectos como funcionamiento familiar, felicidad, maltrato, depresión, riesgo nutricional y discriminación. El apoyo social percibido se evaluó mediante el Cuestionario de Estudio de Desenlaces Médicos de Apoyo Social (Medical Outcomes Study- Social Support Survey (MOS)). La muestra fue de 1452 personas seleccionadas por muestreo probabilístico por conglomerado, bietápico, en las ciudades de Medellín, Barranquilla y Pasto (Colombia). Se realizó análisis univariado, bivariado y multivariado, se calcularon medidas estadísticas y epidemiológicas. Se consideraron asociaciones estadísticas aquellas con un valor $p$ inferior a 5\%. Resultados: los adultos mayores participantes percibieron un apoyo social adecuado en sus diferentes dimensiones: $92 \%$ emocional, 93,5\% instrumental, 92\% interacción social positiva y 93\% apoyo afectivo, y $91,9 \%$ en el índice global de apoyo. Percibir buen apoyo social fue una condición predominante en los adultos mayores: con pareja, sin riesgo de depresión, afiliados al Sistema General de Seguridad Social en Salud, que se sienten felices, que conviven con al menos dos familiares, que perciben un adecuado funcionamiento familiar, no reciben malos tratos al interior de su familia, tienen al menos cuatro amigos cercanos, no se sienten discriminados, que están acompañados y tienen alguien que los cuidan permanentemente.

Palabras clave: Apoyo Social, Interacción Social, Adulto Mayor, Relaciones Familiares.

\section{Abstract}

Objective: Identify the factors that explain the social support components of the elderly. Methods: quantitative, cross-sectional, analytical study of a source of primary information, obtained from surveys of older adults. The 
Profesional en Gerencia de Sistemas de Información en Salud. Integrante del Grupo de Investigación Epidemiología y Bioestadística, Escuela de Graduados, Universidad CES, Medellín, Colombia.

2. Doctora en Demografía. Magister en Epidemiología. Magister en Salud Pública. Administradora de Empresas. Integrante del Grupo de Investigación Epidemiología y Bioestadística, Facultad de Medicina, Universidad CES, Medellín, Colombia.

3. Doctora en Epidemiología. Magister en Epidemiología. Estadística e Informática. Integrante del Grupo de Investigación Observatorio de la Salud Pública, Escuela de Graduados, Universidad CES, Medellín, Colombia.

4. Magister en Epidemiología. Psicóloga. Integrante del Grupo de Investigación Psicología, Salud y Sociedad. Facultad de Psicología, Universidad CES, Medellín, Colombia.

5. Estudiante de Doctorado en Epidemiología y Bioestadística. Profesional en Gerencia de Sistemas de Información en Salud. Grupo de Investigación Epidemiología y Bioestadística, Escuela de Graduados, Universidad CES, Medellín, Colombia.

6. Estudiante de Doctorado en Epidemiología y Bioestadística. Magister en Epidemiología. Especialista en Pedagogía y Docencia Universitaria. Fisioterapeuta. Integrante del Grupo de

http://dx.doi.org/10.21615/cesp.13.2.10 sample was 1452 people selected by probabilistic sampling by cluster, two-stage. In Medellín, Barranquilla and Pasto. To measure the perceived social support, the Medical. Results Study Social Support Survey was applied. Univariate, bivariate and multivariate analyzes were performed, statistical and epidemiological measures were calculated. Statistical associations considered with a $p$ value of less than $5 \%$ were considered. Results: In relation to the social support components of the elderly, with an adequate condition: emotional support (92\%); instrumental support (93.2\%); positive social interaction (92.2\%); affective support (93.2\%) and in the total evaluation (91.9\%). The city of Pasto was the one that selected the lowest percentages, in the different components. Perceiving good social support was a predominant condition in older adults: with a partner, without depression, affiliated with the General System of Social Security in Health, happy (through the Lima happiness scale), who live with at least two relatives, with proper family functioning, they do not perceive ill-treatment within their family, they have at least four close friends, they do not feel discriminated against, they do not leave them constantly alone and they have someone to take care of them permanently. Conclusion: being from the city of Medellín, having a partner, feeling happy, not being alone, as well as not having depression, important factors in each of the components of social support.

Keywords: Social Support, Interpersonal Relations, Older Adult, Aging, Family Relations.

\section{Introducción}

Las dinámicas demográficas actuales han generado cambios en la estructura poblacional, con un aumento acelerado de la población mayor de 60 años, acompañado de la disminución del segmento infantil. Actualmente, a nivel mundial, los adultos mayores representan el 9\% de la población total y se estima que para el año 2050 esta cifra aumentará a 16\% (Organización de Naciones Unidas, 2019). En Colombia, en el último censo realizado en el año 2018, se evidenció que del total de la población, 13,3\% correspondía a personas de 60 años (Departamento Administrativo Nacional de Estadística [DANE], 2018). Por su parte, Cardona y Peláez (2012) señalan algunos retos derivados del envejecimiento poblacional como el aumento de la demanda de atención en el sistema de seguridad social en salud y el incremento de la necesidad de cuidado por parte de las familias. El envejecimiento poblacional genera cambios económicos, sociales y familiares, que requieren acciones para garantizar una adecuada atención y cuidados a la población que envejece (Aroila, 2003; Gómez \& Sabeh, 2001; Palomba, 2002; Riaño, 1991).

El ser humano por naturaleza es sociable y a lo largo de la vida va forjando lazos y vínculos con la familia, los amigos, compañeros y cercanos. Este proceso de socialización se apoya en la necesidad que tienen los individuos de recibir afecto, seguridad, reconocimiento, ayuda o apoyo (Maslow, 1964). Por lo general, el individuo interactúa con su círculo más cercano que es la familia, en principio recibe todo lo que necesita de sus padres, aprende conductas que le permiten socializar y desarrolla un lenguaje que le facilita expresar todo aquello que necesita. De acuerdo con esto, la familia es el primer y más importante lugar donde se forjan los primeros vínculos; interacciones que se requerirán en todas las etapas de la vida.

El apoyo social, entendido como un conjunto de transferencias de ayuda, afecto y opinión entre personas, permite pertenecer a una comunidad y además constituye un soporte económico, material, emocional y cognitivo para afrontar diversos aspectos de la vida (Arias, 2008). El apoyo social en la vejez se concibe como el cuidado, la atención y comunicación necesarios para que las personas en esta etapa puedan 
Pág 146

Investigación Epidemiología y Bioestadística, Escuela de Graduados, Universidad CES, Medellín, Colombia.
Un estudio realizado con población mayor de Colombia señala que la escasez de apoyo social en la vejez repercute negativamente en los diferentes aspectos de la vida de los adultos mayores, además, aumenta los sentimientos negativos de desprotección y soledad (Porras-Juárez et al., 2010). mantener una vida con un nivel mínimo de seguridad y confianza en el medio en el que se encuentran (Esquivel \& García, 2017). Un adecuado apoyo social durante el proceso de envejecimiento sirve de herramienta para continuar participando activamente de la sociedad (Zapata-López, Delgado-Villamizar, \& Cardona-Arango, 2015), y tiene importantes repercusiones en la salud de los adultos mayores; así, por ejemplo, según un estudio realizado por Peláez y Rodríguez (2012), las personas con mayor integración social viven más, tienen mayores probabilidades de sobrevivir a un infarto de miocardio, presentan menores síntomas depresivos, menos recurrencias de cáncer y son menos propensos a padecer enfermedades infecciosas; y estar aislado supone un riesgo para la salud comparable a los riesgos asociados al hábito de fumar, la presión arterial elevada y la obesidad.

Lamentablemente, no en pocos casos, el proceso del envejecimiento está acompañado de deterioro de la salud y las condiciones económicas, de soledad, abandono y exclusión, sumado a prejuicios sociales asociados a esta etapa de la vida relacionados principalmente con la vulnerabilidad, producto de la enfermedad y pérdida de capacidades físicas y mentales, afectando el estado psicológico y social de esta población (García, 2002; Ramos \& Salinas, 2010; Zavala et al., 2006).

Un estudio realizado con población mayor de Colombia señala que la escasez de apoyo social en la vejez repercute negativamente en los diferentes aspectos de la vida de los adultos mayores, además, aumenta los sentimientos negativos de desprotección y soledad (Porras-Juárez et al., 2010). En Colombia, en la ciudad de Pasto, una investigación realizada en el año 2016 identificó que la escasez de apoyo social es una de las principales características asociadas con el maltrato de la población mayor, por lo cual es necesario el fortalecimiento de redes de apoyo en la vejez (Agudelo-Cifuentes et al., 2019). Y en la ciudad de Medellín, Cardona, Estrada y Agudelo (2003) encontraron que "los adultos mayores, dentro de su núcleo familiar viven una situación en la que, a pesar de estar acompañados por sus familiares, sus aportes y decisiones no son tenidos en cuenta" (p. 12).

Dada la necesidad de avanzar en el conocimiento sobre la situación del adulto mayor en Colombia y las repercusiones positivas derivadas de una adecuada red de apoyo social; y específicamente, sobre la percepción que tiene el adulto mayor respecto a los diferentes tipos de apoyo y como estos contribuyen a su bienestar, el presente estudio se planteó con el objetivo de identificar los factores asociados a las dimensiones del apoyo social del adulto mayor en tres ciudades de Colombia: Medellín, Barranquilla y Pasto.

\section{Método}

Se realizó un estudio empírico analítico, de diseño transversal, en el que se utilizó una fuente de información primaria, obtenida a partir de encuestas realizadas a personas mayores de las ciudades de Medellín, Barranquilla y Pasto (Colombia). La selección de los participantes se realizó mediante un muestreo probabilístico, por conglomerados, bietápico, según la distribución geográfica de la población mayor en Colombia. Las características del muestreo permitieron expandir los resultados a la totalidad de adultos mayores de estas ciudades. Para el cálculo del tamaño de la muestra se utilizó la fórmula de Fleiss para poblaciones finitas, con una confianza del $95 \%$, un error permisible del $5 \%$ y un efecto de diseño del 1,0. Así, se obtuvo una muestra de 1.514 adultos de 60 años y más de edad distribuidos de la siguiente manera: 495 en la ciudad de Medellín, 513 en Barranquilla y 506 en Pasto. 


\section{Instrumentos}

Como técnica de recolección de información se usó un cuestionario diseñado para esta investigación que indagó sobre características demográficas, familiares y sociales de los participantes; también incluyó algunas escalas de medición validadas, que permitieron evaluar diversos aspectos como: apoyo social, funcionamiento familiar, felicidad, maltrato, depresión, riesgo nutricional y discriminación (Tabla 1)

Tabla 1. Preguntas y Escalas de medición incluidas en el cuestionario de recolección de datos

\begin{tabular}{|c|c|}
\hline Aspectos/Características & Pregunta/Escalas \\
\hline Sociodemográficas & $\begin{array}{l}\text { Se indagó por el sexo, el estado civil, el nivel educativo, el rol en } \\
\text { el hogar, si había recibido o no algún ingreso económico durante } \\
\text { el mes anterior a la encuesta y se consultó si había consumido } \\
\text { cigarrillo en el pasado o en la actualidad, y afiliación al Sistema } \\
\text { General de Seguridad Social en Salud (SGSSS) }\end{array}$ \\
\hline Funcionamiento cognitivo & $\begin{array}{l}\text { Escala Mini-Examen de Estado Mental (MMSE) (Folstein \& } \\
\text { McHugh, 1975) }\end{array}$ \\
\hline $\begin{array}{l}\text { Satisfacción situación } \\
\text { económica }\end{array}$ & ¿Qué tan satisfecho está con su situación económica actual? \\
\hline Participación comunitaria & ¿Cuál es la principal organización comunitaria en la que participa? \\
\hline Satisfacción salud & ¿Qué tan satisfecho está con su salud? \\
\hline Discapacidad & $\begin{array}{l}\text { ¿Tiene alguna discapacidad? } \\
\text { 1. Para escuchar } \\
\text { 2. Para ver } \\
\text { 3. Para moverse (caminar, correr) } \\
\text { 4. Para hablar } \\
\text { 5. Para agarrar }\end{array}$ \\
\hline Enfermedades de base & $\begin{array}{l}\text { ¿Ha sido diagnosticado con alguna de las siguientes condiciones } \\
\text { médicas? }\end{array}$ \\
\hline Riesgo nutricional & Nutrition Screening Initiative Determine Checklist (NSI) \\
\hline Satisfacción calidad de vida & ¿Qué tan satisfecho está con su calidad de vida? \\
\hline Felicidad & Escala de Felicidad de Lima (Alarcón, 2006) \\
\hline Pensamientos suicidas & ¿Ha pensado en atentar contra su vida? \\
\hline Riesgo de depresión & $\begin{array}{l}\text { Escala de Depresión Geriátrica Abreviada de Yesavage (Sheik \& } \\
\text { Yesavage, 1986). }\end{array}$ \\
\hline Número de familiares & $N^{\circ}$ de familiares cercanos a usted \\
\hline Tipo de familia & $\begin{array}{l}\text { Personas que conforman la familia: } \\
1 \text { Nuclear (padres-hijos) } \\
2 \text { Extensa (padres-hijos-otros parientes) } \\
3 \text { Reconstruida (padres-hijos-otros no parientes) } \\
4 \text { Monoparental }\end{array}$ \\
\hline Le han dejado solo & ¿Le han dejado solo por largos periodos de tiempo? \\
\hline Malos tratos entre familiares & ¿Ha observado malos tratos entre los miembros de su familia? \\
\hline Cuidador & ¿Tiene usted quien lo cuide? \\
\hline Funcionamiento familiar & Escala APGAR Familiar (Smilkstein, 1978). \\
\hline Maltrato & $\begin{array}{l}\text { Escala Geriátrica de Maltrato (Giraldo-Rodríguez \& Rosas- } \\
\text { Carrasco, 2013). }\end{array}$ \\
\hline Número de amigos & $N^{\circ}$ de amigos cercanos a usted \\
\hline Apoyo social & $\begin{array}{l}\text { Medical Outcomes Study Social Support Survey (MOS-SS) } \\
\text { (Sherbourne \& Stewart, 1991). }\end{array}$ \\
\hline Discriminación & $\begin{array}{l}\text { Escala de discriminación en la vida cotidiana (Campo-Arias et al., } \\
\text { 2015) }\end{array}$ \\
\hline
\end{tabular}


Escala Mini-Examen de Estado Mental (MMSE, por sus siglas en inglés) Se utilizó para evaluar el estado cognitivo de los adultos mayores. Está conformada por 11 preguntas que analizan algunas áreas del funcionamiento cognitivo: orientación, registro, atención, cálculo, memoria y lenguaje. El MMSE ha sido validado y utilizado extensamente, tanto en la práctica como en la investigación clínica desde su creación por Folstein y McHugh en 1975 (Icaza \& Albala, 1999).

Medical Outcomes Study Social Support Survey (MOS-SS) (Sherbourne \& Stewart, 1991). Se utilizó para medir el apoyo social percibido por los adultos mayores. Evalúa las dimensiones que componen la red de apoyo social: la estructural, que indaga por el número de personas que conforman la red de apoyo social (amigos y familiares) de una persona, y la funcional, que comprende el apoyo social emocional/informacional, con foco en el soporte emocional, orientación y consejos; el apoyo instrumental caracterizado por la conducta o material de apoyo; interacción social positiva, que se refiere a la disponibilidad de personas para realizar actividades divertidas; y apoyo afectivo, enfocado en las posibilidades de brindar y recibir afecto. Para esta investigación fue aplicada la escala MOS-SS validada en Colombia (Arredondo, 2012), la cual presentó una adecuada consistencia interna de 0,941.

Escala de Felicidad de Lima (Alarcón, 2006).

Se utilizó para evaluar la felicidad, entendida como un sentimiento de satisfacción que experimenta una persona. Cuenta con 27 ítems tipo Likert que evalúan cuatro dimensiones o factores de la felicidad: Sentido positivo de la vida, Satisfacción con la vida, Realización personal y Alegría de vivir. Previas validaciones han mostrado que es una escala confiable, con una consistencia interna aceptable, alfa de Cronbach de 0,84 (Árraga Barrios \& Sánchez Villarroel, 2011).

Escala APGAR familiar.

Construida por Smilkestein en 1978, es un instrumento de cinco preguntas mediante las que se evalúa la percepción del nivel de funcionamiento de la unidad familiar, a partir de algunas funciones básicas: Adaptación, entendida como la capacidad de los miembros para utilizar recursos tanto internos como externos en la solución de situaciones de crisis; Participación, o cooperación de cada integrante de la familia en la toma de decisiones y en la apropiación de responsabilidades que busquen el mantenimiento de la familia; Gradiente de recurso personal, que hace referencia al nivel de madurez de los componentes familiares por medio del apoyo mutuo; Afecto y muestras de cariño entre los integrantes de la familia; y, finalmente, Recursos o necesidades tanto físicos como emocionales de los miembros de la familia que requieren compartir, ya sea ingresos o espacios (Bellón, Delgado, Luna del Castillo, \& Lardelli, 1996). Al sumar las cinco respuestas el puntaje fluctúa entre 0 y 20 , e indica diversos niveles de funcionamiento familiar: Normal, Disfunción leve, Disfunción moderada y Disfunción severa.

Escala Geriátrica de Maltrato (Giraldo-Rodríguez \& Rosas-Carrasco, 2013). Instrumento diseñado para la detección de cinco tipos de maltrato en personas mayores (físico, psicológico, económico, sexual y negligencia). Fue validada en adultos mayores mexicanos, mostrando valores de consistencia interna para sus cinco dimensiones, superiores a 0,80.

Escala de Depresión Geriátrica Abreviada de Yesavage (Sheik \& Yesavage, 1986).

Evalúa la presencia de riesgo de depresión en población de adultos mayores. Es un cuestionario breve en el cual se le pide al individuo responder 15 preguntas en torno 
Pág 149

a su posible experiencia de síntomas cognoscitivos de un episodio depresivo mayor, durante la semana anterior a la fecha en que se administra, con un patrón de respuesta dicotómica para facilitar su diligenciamiento. De acuerdo con las respuestas y ponderación de resultados, la persona evaluada es clasificada: sin riesgo de depresión, con riesgo de depresión leve y con riesgo de depresión alto.

Nutrition Screening Initiative Determine Checklist (NSI).

Este instrumento permite detectar factores de riesgo asociados a la malnutrición en adultos mayores; fue desarrollado por la Asociación Americana de Dietética, la Academia Americana de Médicos de Familia y el Consejo Nacional sobre Envejecimiento (Beck et al., 1999). Cuenta con diez ítems asociados con el bienestar nutricional. Las puntuaciones finales permiten clasificar a la persona evaluada en dos categorías: con riesgo o sin riesgo nutricional.

Escala de Discriminación en la Vida Cotidiana (EDVC) (Campo-Arias et al., 2015). Consta de 10 preguntas que evalúan situaciones de discriminación en las diferentes etapas de la vida, entendiendo por discriminación, al conjunto de comportamientos y normas que vulneran los derechos de una persona que suele hacer parte de un grupo con determinadas condiciones que socialmente han sido valoradas como negativas y que le ponen en desventaja. Esta escala permite la exploración de experiencias de esta índole por causa de diversas variables.

\section{Procedimiento y aspectos éticos}

Para la selección de los participantes se partió de un marco muestral de todas las manzanas $^{1}$ de las tres ciudades que forman parte del estudio, y se encuestaron a todos los adultos mayores que habitaban las viviendas de las manzanas seleccionadas. La encuesta y los instrumentos fueron aplicados en los hogares, por encuestadores capacitados y estandarizados en el uso de los instrumentos utilizados. Previo a la recolección de los datos, se realizó una prueba piloto con el propósito de validar la metodología del estudio y la estandarización de los encuestadores (10 adultos mayores, por cada uno de los encuestadores). Quienes hicieron parte de la prueba piloto no hicieron parte de la muestra. Se establecieron como criterios de inclusión una edad mayor a 60 años y más, ser residente del área urbana y aceptar participar y firmar el consentimiento informado. Como criterio de exclusión, se determinó el deterioro cognitivo de acuerdo con la prueba tamiz Mini Examen de Estado Mental (MMSE) o que estuvieran bajo efecto de sustancias psicoactivas. El MMSE fue aplicado a todas las personas que cumplieron criterios de inclusión, en caso de obtener un puntaje superior a 13, se continuó con la aplicación del cuestionario de recolección de datos; en caso contrario, se agradeció a la persona voluntaria y no se aplicó el cuestionario.

Para el desarrollo de la investigación se contó con la aprobación del comité operativo de investigación de la Facultad de Medicina y del comité de ética de la investigación en seres humanos de la Universidad CES. En conformidad con la Resolución 8430 de 1993, esta investigación se clasificó como de riesgo mínimo, ya que no se realizaron tratamientos ni procedimientos físicos, pero se incluyeron preguntas a los adultos mayores sobre temas sensibles, como aquellas relacionadas con la depresión, el suicidio y el maltrato. Cada adulto mayor fue informado acerca del derecho a retirarse de la investigación en el momento en que lo deseara. La investigación contó con profesionales de psicología dispuestos en caso de que, derivado de las preguntas dirigidas, algún participante presentara una reacción que requiriera tratamiento 
Pág 150

Los resultados de la presente investigación permitieron identificar que $92 \%$ de los adultos mayores participantes percibieron un adecuado apoyo emocional, siendo mayor el porcentaje en la ciudad de Barranquilla (97\%), seguido de Medellín (95\%). profesional. Adicionalmente, se brindaron rutas de atención a los participantes para que pudieran activarlas en caso de que experimentaran situaciones de vulnerabilidad.

\section{Análisis de datos}

Para el análisis univariado se contemplaron las características demográficas, de salud física y mental y de relaciones familiares y sociales, de los adultos mayores de las tres ciudades. Para los análisis bivariados se tomó como variable dependiente el apoyo social, con el fin de identificar las características demográficas, de salud física y mental y de relaciones familiares que pudieran estar asociadas a la escasez de apoyo social; y se utilizó el estadístico chi cuadrado (X.2), además se calcularon las razones de prevalencias crudas; las variables con valores de $p<0,25$ se consideraron para ser utilizadas en el modelo multivariado, y para sacar las medidas ajustadas (razón de prevalencias y su respectivo intervalo de confianza), valores de $p<0,05$ se consideraron estadísticamente significativas. El procesamiento de los datos se realizó por medio del paquete SPSS versión 21 (SPSS Inc; Chicago, Illinois, USA) licencia de la Universidad CES.

\section{Resultados}

\section{Características sociodemográficas de la población mayor}

En el presente estudio participaron 1452 adultos mayores, distribuidos de la siguiente forma: residentes en la ciudad de Medellín 31,7\%, Barranquilla 34,3\% y Pasto 34\%. La edad promedio de los adultos mayores participantes fue de 70,6 años (IC: 70,3; 71,1); el mayor porcentaje (73\%) de adultos mayores, según los rangos de edad establecidos por la Organización Mundial de la Salud, fue el de los jóvenes (entre 60 y 74 años), seguido de los viejos (entre 75 y 89 años) con $25,2 \%$, en menor porcentaje los longevos (entre 90 y 99 años) con 1,7\%, y tan solo un adulto mayor era centenario; las mujeres representaron $65,6 \%$ y los hombres 34,4\%. 50,3\% tenían pareja (casado o en unión libre), a diferencia del $49,7 \%$ restante (soltero, separado, divorciado o viudo); la mayoría de los adultos mayores participantes $(66,5 \%)$ vivía en estrato socioeconómico bajo (estrato 1 o 2), 30,9\% en estrato medio (estrato 3 o 4) y 2,6\% en estrato alto (estrato $5 \circ 6$ ); y 81,2\% tenían estudios de primaria o secundaria.

En relación con la conformación de la familia, 42,4\% de los adultos mayores pertenecían a una familia nuclear y $42 \%$ extensa; además, $6,3 \%$ de los adultos mayores reportaron convivir con familias con disfuncionalidad severa y $8,2 \%$ en familias con disfuncionalidad moderada, según el APGAR familiar.

En cuanto a la presencia de depresión, 4,3\% de los participantes presentaban un riesgo alto y $9,1 \%$ un riesgo bajo; por último, 9,7\% de los adultos mayores reportó ser víctima de algún tipo de maltrato.

\section{Apoyo social de las personas mayores}

Los resultados de la presente investigación permitieron identificar que 92\% de los adultos mayores participantes percibieron un adecuado apoyo emocional, siendo mayor el porcentaje en la ciudad de Barranquilla (97\%), seguido de Medellín (95\%). La percepción de adecuado apoyo instrumental se presentó en 93,5\% de los adultos mayores participantes; interacción social positiva en 92\% y apoyo afectivo en 93\%. La ciudad de Barranquilla fue la que mostró las cifras más altas de percepción de adecuado apoyo social en sus diferentes dimensiones, seguido de la ciudad de Medellín y en último lugar Pasto. 


\section{Apoyo emocional}

Se observó que las variables estado civil, recepción de ingresos económicos en el mes anterior, presencia de pensamientos suicidas, riesgo de depresión alto, satisfacción con su calidad de vida, satisfacción con su salud, satisfacción con su situación económica, felicidad y discriminación, presentaron asociación significativa con la percepción de un adecuado apoyo emocional. Estas variables fueron sometidas a un análisis ajustado para controlar posibles factores confusores y algunas mantuvieron su significancia estadística de modo que los adultos mayores que tenían pareja presentaron mayor prevalencia respecto a quienes no tenían pareja (RPa: 4,2; IC95\%: 2,2; 8), así como quienes no habían recibido ingresos económicos en el mes anterior a la recolección de información (RPa: 2,0; IC95\%: 1,2; 3,4) y las personas mayores sin riesgo de depresión, tuvieron mayor probabilidad de percibir un adecuado apoyo emocional (RPa: 3,0; IC95\%: 1,3; 6,6) (Ver Tabla 2).

Tabla 2. Razones de prevalencia crudas y ajustadas de la dimensión apoyo emocional, de los adultos mayores de tres ciudades de Colombia, 2016

\begin{tabular}{|c|c|c|c|c|c|c|c|c|c|c|c|c|c|}
\hline & \multirow{3}{*}{ Variable } & \multicolumn{4}{|c|}{ Apoyo emocional } & \multirow{3}{*}{$p$} & \multirow{3}{*}{$R P c$} & \multirow{3}{*}{$L I$} & \multirow{3}{*}{$L S$} & \multirow{3}{*}{$p$} & \multirow{3}{*}{$R P a$} & \multirow{3}{*}{$L i$} & \multirow{3}{*}{ Ls } \\
\hline & & \multicolumn{2}{|c|}{ Adecuado } & \multicolumn{2}{|c|}{ Escaso } & & & & & & & & \\
\hline & & $N$ & $\%$ & $N$ & $\%$ & & & & & & & & \\
\hline \multirow{3}{*}{ Ciudad } & Medellín & 438 & 32,7 & 24 & 20,7 & \multirow{3}{*}{0,00} & 3,4 & 2,1 & 5,4 & \multirow{3}{*}{0,0} & 3,2 & 1,7 & 6,1 \\
\hline & Barranquilla & 481 & 35,9 & 15 & 12,9 & & 5,9 & \multirow[t]{2}{*}{3,3} & 10,4 & & 2,0 & 1,0 & 4,1 \\
\hline & Pasto & 419 & 31,3 & 77 & 66,4 & & 1,0 & & & & 1,0 & & \\
\hline \multirow{2}{*}{ Sexo } & Hombre & 461 & 34,5 & 40 & 34,5 & \multirow{2}{*}{0,99} & 1,0 & & & \multirow{2}{*}{0,3} & & & \\
\hline & Mujer & 877 & 65,5 & 76 & 65,5 & & 1,0 & 0,7 & 1,5 & & 1,4 & 0,8 & 2,4 \\
\hline \multirow{2}{*}{ Estado civil } & Sin pareja & 636 & 47,5 & 86 & 74,1 & \multirow{2}{*}{0,00} & 1,0 & & & \multirow{2}{*}{0,0} & 1,0 & & \\
\hline & Con pareja & 702 & 52,5 & 30 & 25,9 & & 3,2 & 2,1 & 4,9 & & 4,2 & 2,2 & 8,0 \\
\hline \multirow{4}{*}{ Nivel educativo } & Ninguno & 124 & 9,3 & 16 & 13,8 & \multirow{4}{*}{0,05} & 1,0 & & & \multirow{4}{*}{1,0} & & & \\
\hline & Primaria & 702 & 52,5 & 70 & 60,3 & & 1,3 & 0,7 & 2,3 & & 1,1 & 0,5 & 2,3 \\
\hline & Secundaria & 386 & 28,8 & 23 & 19,8 & & 2,2 & 1,1 & 4,2 & & 1,3 & 0,5 & 3,0 \\
\hline & Terciaria & 126 & 9,4 & 7 & 6 & & 2,3 & 0,9 & 5,8 & & 1,1 & 0,3 & 3,3 \\
\hline \multirow{5}{*}{ Rol en el hogar } & Jefe de hogar & 838 & 62,6 & 75 & 65,2 & \multirow{5}{*}{0,21} & 1,4 & 0,6 & 3,2 & & 0,5 & 0,2 & 1,8 \\
\hline & Cónyuge del jefe de hogar & 249 & 18,6 & 13 & 11,3 & & 2,4 & 0,9 & 6,4 & & 0,4 & 0,1 & 1,7 \\
\hline & Padre del jefe de hogar & 149 & 11,1 & 13 & 11,3 & & 1,5 & 0,6 & 3,8 & 0,0 & 1,6 & 0,4 & 6,3 \\
\hline & Hermano del jefe de hogar & 47 & 3,5 & 7 & 6,1 & & 0,9 & 0,3 & 2,6 & & 0,3 & 0,1 & 1,2 \\
\hline & Otro & 55 & 4,1 & 7 & 6,1 & & 1,0 & & & & 1,0 & & \\
\hline Ingreso económico & Si & 739 & 55,3 & 82 & 70,7 & R & 1,0 & & & & 1,0 & & \\
\hline mes anterior & No & 598 & 44,7 & 34 & 29,3 & U,U & 2,0 & 1,3 & 3,0 & U,U & 2,0 & 1,2 & 3,4 \\
\hline & Antes & 441 & 33 & 36 & 31 & & 1,0 & & & & 1,0 & & \\
\hline Cigarrillo & Ahora & 122 & 9,1 & 18 & 15,5 & 0,09 & 0,6 & 0,3 & 1,0 & 0,4 & 0,6 & 0,3 & 1,3 \\
\hline & Nunca & 774 & 57,9 & 62 & 53,4 & & 1,0 & 0,7 & 1,6 & & 1,1 & 0,6 & 1,9 \\
\hline Pensar en atentar & No & 1264 & 94,5 & 96 & 82,8 & R & 3,6 & 2,1 & 6,1 & 12 & 1,6 & 0,8 & 3,2 \\
\hline contra la vida & $\mathrm{Si}$ & 74 & 5,5 & 20 & 17,2 & 0,00 & 1,0 & & & 0,2 & 1,0 & & \\
\hline & No depresión & 1205 & 90,1 & 55 & 47,4 & & 11,8 & 6,6 & 21,1 & & 3,0 & 1,3 & 6,6 \\
\hline Riesgo de depresión & Probable depresión & 92 & 6,9 & 39 & 33,6 & 0,00 & 1,3 & 0,7 & 2,4 & 0,0 & 0,8 & 0,4 & 1,9 \\
\hline & Depresión establecida & 41 & 3,1 & 22 & 19 & & 1,0 & & & & 1,0 & & \\
\hline Satisfacción calidad & Insatisfecho & 84 & 6,3 & 42 & 36,2 & חمी & 1,0 & & & 03 & 1,0 & & \\
\hline de vida & Satisfecho & 1250 & 93,7 & 74 & 63,8 & & 8,4 & 5,4 & 13,1 & 0 & 1,4 & 0,7 & 2,7 \\
\hline
\end{tabular}




\begin{tabular}{|c|c|c|c|c|c|c|c|c|c|c|c|c|c|}
\hline & \multirow{3}{*}{ Variable } & \multicolumn{4}{|c|}{ Apoyo emocional } & \multirow{3}{*}{$p$} & \multirow{3}{*}{$R P c$} & \multirow{3}{*}{$L I$} & \multirow{3}{*}{$L S$} & \multirow{3}{*}{$p$} & \multirow{3}{*}{$R P a$} & \multirow{3}{*}{$L i$} & \multirow{3}{*}{ Ls } \\
\hline & & \multicolumn{2}{|c|}{ Adecuado } & \multicolumn{2}{|c|}{ Escaso } & & & & & & & & \\
\hline & & $N$ & $\%$ & $N$ & $\%$ & & & & & & & & \\
\hline \multirow{2}{*}{ Satisfacción salud } & Insatisfecho & 169 & 12,7 & 51 & 44 & \multirow{2}{*}{0,00} & 1,0 & \multirow[b]{2}{*}{3,6} & \multirow[b]{2}{*}{8,1} & \multirow{2}{*}{0,5} & 1,0 & & \\
\hline & Satisfecho & 1165 & 87,3 & 65 & 56 & & 5,4 & & & & 1,2 & 0,7 & 2,3 \\
\hline \multirow{2}{*}{$\begin{array}{l}\text { Satisfacción } \\
\text { situación económica }\end{array}$} & Insatisfecho & 369 & 27,7 & 79 & 68,1 & \multirow{2}{*}{0,00} & 1,0 & & & \multirow{2}{*}{0,0} & 1,0 & & \\
\hline & Satisfecho & 965 & 72,3 & 37 & 31,9 & & 5,6 & 3,7 & 8,4 & & 1,9 & 1,1 & 3,3 \\
\hline \multirow{2}{*}{ Felicidad } & Feliz & 1165 & 88,3 & 42 & 36,2 & \multirow{2}{*}{0,00} & 13,3 & \multirow[t]{2}{*}{8,8} & \multirow[t]{2}{*}{20,2} & \multirow{2}{*}{0,0} & 4,4 & 2,5 & 7,7 \\
\hline & Infeliz & 154 & 11,7 & 74 & 63,8 & & 1,0 & & & & 1,0 & & \\
\hline \multirow{2}{*}{ Discriminación } & No discriminado & 1187 & 88,8 & 64 & 55,2 & \multirow{2}{*}{0,00} & 6,4 & \multirow[t]{2}{*}{4,3} & \multirow[t]{2}{*}{9,6} & \multirow{2}{*}{0,0} & 2,5 & 1,5 & 4,2 \\
\hline & Discriminado & 150 & 11,2 & 52 & 44,8 & & 1,0 & & & & 1,0 & & \\
\hline \multirow{2}{*}{ Le han dejado solo } & $\mathrm{Si}$ & 121 & 9 & 30 & 25,9 & \multirow{2}{*}{0,00} & 1,0 & & & \multirow{2}{*}{0,0} & 1,0 & & \\
\hline & No & 1217 & 91 & 86 & 74,1 & & 3,5 & 2,2 & 5,5 & & 4,9 & 2,6 & 9,2 \\
\hline
\end{tabular}

*RPc: razón de prevalencias cruda; RPa: razón de prevalencias ajustada; Li: límite inferior; Ls: límite superior

\section{Apoyo Instrumental}

Respecto a la dimensión de apoyo instrumental, las variables que presentaron asociación estadística fueron: estado civil, ausencia de discapacidad para escuchar, ausencia de riesgo nutricional, recepción de subsidio o ayuda económica, no haber sido dejado solo y no experimentar situaciones de discriminación ni de maltrato. Al realizar el análisis multivariado para ajustar las estimaciones, las variables que mantuvieron su significancia estadística fueron: tener pareja (RPa: 2,8; IC95\%: 1,7; 4,8), no haber sido dejado solo por largos periodos de tiempo (RPa: 3,8; IC95\%: 2,2; 6,8) y no haber sido maltratado (RPa: 0,5; IC95\%: 0,3; 0,8) (Ver Tabla 3).

Tabla 3. Razones de prevalencia crudas y ajustadas de la dimensión apoyo instrumental de los adultos mayores de tres ciudades de Colombia, 2016

\begin{tabular}{|c|c|c|c|c|c|c|c|c|c|c|c|c|c|}
\hline \multirow{2}{*}{\multicolumn{2}{|c|}{ Variable }} & \multicolumn{4}{|c|}{ Apoyo instrumental } & \multirow{3}{*}{$p$} & \multirow{3}{*}{$R P c$} & \multirow{3}{*}{$L I$} & \multirow{3}{*}{$L S$} & \multirow{3}{*}{$p$} & \multirow{3}{*}{$R P a$} & \multirow{3}{*}{$L i$} & \multirow{3}{*}{ Ls } \\
\hline & & \multicolumn{2}{|c|}{ Adecuado } & \multicolumn{2}{|c|}{ Escaso } & & & & & & & & \\
\hline & & $N$ & $\%$ & $N$ & $\%$ & & & & & & & & \\
\hline \multirow{3}{*}{ Ciudad } & Medellín & 439 & 32,3 & 23 & 24,2 & \multirow{3}{*}{0,00} & 2,2 & 1,3 & 3,7 & \multirow{3}{*}{0,0} & 2,5 & 1,4 & 4,6 \\
\hline & Barranquilla & 476 & 35 & 20 & 21,1 & & 2,8 & 1,6 & 4,7 & & 1,7 & 0,9 & 3,1 \\
\hline & Pasto & 444 & 32,7 & 52 & 54,7 & & 1,0 & & & & 1,0 & & \\
\hline \multirow{2}{*}{ Sexo } & Hombre & 472 & 34,7 & 29 & 30,5 & \multirow{2}{*}{0,41} & 1,0 & & & \multirow{2}{*}{0,7} & 1,0 & & \\
\hline & Mujer & 887 & 65,3 & 66 & 69,5 & & 0,8 & 0,5 & 1,3 & & 0,9 & 0,5 & 1,5 \\
\hline \multirow{2}{*}{ Estado civil } & Sin pareja & 652 & 48 & 70 & 73,7 & \multirow{2}{*}{0,00} & 1,0 & & & \multirow{2}{*}{0,0} & 1,0 & & \\
\hline & Con pareja & 707 & 52 & 25 & 26,3 & & 3,0 & 1,9 & 4,9 & & 2,8 & 1,7 & 4,8 \\
\hline \multirow{4}{*}{ Nivel educativo } & Ninguno & 124 & 9,1 & 16 & 16,8 & \multirow{4}{*}{0,05} & 1,0 & & & \multirow{4}{*}{0,6} & 1,0 & & \\
\hline & Primaria & 719 & 52,9 & 53 & 55,8 & & 1,8 & 1,0 & 3,2 & & 1,5 & 0,8 & 2,8 \\
\hline & Secundaria & 389 & 28,6 & 20 & 21,1 & & 2,5 & 1,3 & 5,0 & & 1,5 & 0,7 & 3,3 \\
\hline & Terciaria & 127 & 9,3 & 6 & 6,3 & & 2,7 & 1,0 & 7,2 & & 1,9 & 0,6 & 5,7 \\
\hline \multirow{2}{*}{$\begin{array}{l}\text { Ingreso económico } \\
\text { mes anterior }\end{array}$} & $\mathrm{Si}$ & 759 & 55,9 & 62 & 65,3 & \multirow{2}{*}{0,08} & 1,0 & & & \multirow{2}{*}{0,1} & 1,0 & & \\
\hline & No & 599 & 44,1 & 33 & 34,7 & & 1,5 & 1,0 & 2,3 & & 1,5 & 0,9 & 2,6 \\
\hline \multirow{2}{*}{$\begin{array}{l}\text { Afiliado al SGSS en } \\
\text { Salud }\end{array}$} & $\mathrm{Si}$ & 1321 & 97,2 & 89 & 93,7 & \multirow{2}{*}{0,06} & 2,3 & 1,0 & 5,7 & \multirow{2}{*}{0,1} & 2,3 & 0,9 & 6,3 \\
\hline & No & 38 & 2,8 & 6 & 6,3 & & 1,0 & & & & 1,0 & & \\
\hline \multirow{2}{*}{$\begin{array}{l}\text { Discapacidad para } \\
\text { escuchar }\end{array}$} & No & 1169 & 86,2 & 73 & 76,8 & \multirow{2}{*}{0,01} & 1,9 & 1,1 & 3,1 & & 1,1 & 0,6 & 2,0 \\
\hline & $\mathrm{Si}$ & 187 & 13,8 & 22 & 23,2 & & 1,0 & & & 0,1 & 1,0 & & \\
\hline
\end{tabular}




\begin{tabular}{|c|c|c|c|c|c|c|c|c|c|c|c|c|c|}
\hline & & \multicolumn{4}{|c|}{ Apoyo instrumental } & \multirow{3}{*}{$p$} & \multirow{3}{*}{$R P c$} & \multirow{3}{*}{ LI } & \multirow{3}{*}{$L S$} & \multirow{3}{*}{$p$} & \multirow{3}{*}{$R P a$} & \multirow{3}{*}{$L i$} & \multirow{3}{*}{ Ls } \\
\hline \multirow{2}{*}{\multicolumn{2}{|c|}{ Variable }} & \multicolumn{2}{|c|}{ Adecuado } & \multicolumn{2}{|c|}{ Escaso } & & & & & & & & \\
\hline & & $N$ & $\%$ & $N$ & $\%$ & & & & & & & & \\
\hline \multirow{2}{*}{$\begin{array}{l}\text { Hipertensión } \\
\text { Arterial }\end{array}$} & No & 665 & 48,9 & 53 & 55,8 & \multirow{2}{*}{0,20} & 0,8 & 0,5 & 1,2 & \multirow{2}{*}{0,2} & 0,7 & 0,5 & 1,2 \\
\hline & Si & 694 & 51,1 & 42 & 44,2 & & 1,0 & & & & 1,0 & & \\
\hline \multirow{3}{*}{ Riesgo nutricional } & Sin riesgo & 565 & 41,8 & 23 & 24,5 & \multirow{3}{*}{0,00} & 3,1 & 1,9 & 5,2 & \multirow{3}{*}{0,1} & 1,9 & 1,0 & 3,4 \\
\hline & Riesgo moderado & 377 & 27,9 & 19 & 20,2 & & 2,5 & 1,5 & 4,3 & & 1,8 & 1,0 & 3,3 \\
\hline & Riesgo alto & 411 & 30,4 & 52 & 55,3 & & 1,0 & & & & 1,0 & & \\
\hline \multirow{2}{*}{$\begin{array}{l}\text { Recibe subsidio o } \\
\text { ayuda económica }\end{array}$} & $\mathrm{Si}$ & 303 & 22,3 & 36 & 37,9 & \multirow{2}{*}{0,00} & 1,0 & & & \multirow{2}{*}{0,5} & 1,0 & & \\
\hline & No & 1054 & 77,7 & 59 & 62,1 & & 2,1 & 1,4 & 3,3 & & 1,2 & 0,7 & 2,0 \\
\hline \multirow{2}{*}{ Discriminación } & No discriminado & 1183 & 87,1 & 68 & 71,6 & \multirow{2}{*}{0,00} & 2,7 & 1,7 & 4,3 & \multirow{2}{*}{0,3} & 1,4 & 0,8 & 2,4 \\
\hline & Discriminado & 175 & 12,9 & 27 & 28,4 & & 1,0 & & & & 1,0 & & \\
\hline \multirow{2}{*}{ Le han dejado solo } & Si & 123 & 9,1 & 28 & 29,5 & \multirow{2}{*}{0,00} & 1,0 & & & \multirow{2}{*}{0,0} & 1,0 & & \\
\hline & No & 1236 & 90,9 & 67 & 70,5 & & 4,2 & 2,6 & 6,8 & & 3,8 & 2,2 & 6,8 \\
\hline \multirow{2}{*}{ Maltrato } & No & 1242 & 91,8 & 65 & 69,9 & \multirow{2}{*}{0,00} & 1,0 & & & \multirow{2}{*}{0,0} & 1,0 & & \\
\hline & $\mathrm{Si}$ & 111 & 8,2 & 28 & 30,1 & & 4,8 & 3,0 & 7,8 & & 0,5 & 0,3 & 0,8 \\
\hline
\end{tabular}

*RPc: razón de prevalencias cruda; RPa: razón de prevalencias ajustada; Li: límite inferior; Ls: límite superior

\section{Interacción social positiva}

Al analizar la interacción social positiva de las personas mayores, de acuerdo con las características sociodemográficas, de salud física y mental, y de relaciones familiares, se identificó una estrecha relación en gran número de éstas. Sin embargo, se realizó un análisis multivariado para ajustar por posibles factores de confusión y las características que mostraron asociación significativa con la interacción social positiva fueron: tener pareja (RPa: 4,1; IC95\%: 1,8; 9,4); no presentar riesgo de depresión (RPa: 3,6; IC95\%: 1,5; 8,7); percibir un funcionamiento familiar normal (RPa: 11,2; IC95\%: 5,0; 25,4); no haber sido dejado solo por largos periodos de tiempo (RPa: 2,6; IC95\%: 1,2; 5,7) y tener quien lo cuide (RPa: 2,7; IC95\%: 1,0; 7,2). (Ver Tabla 4).

Tabla 4. Razones de prevalencia crudas y ajustadas de la dimensión interacción social positiva de los adultos mayores de tres ciudades de Colombia, 2016

\begin{tabular}{|c|c|c|c|c|c|c|c|c|c|c|c|c|c|}
\hline & \multirow{3}{*}{ Variable } & \multicolumn{4}{|c|}{ Interacción social positiva } & \multirow{3}{*}{$p$} & \multirow{3}{*}{$R P c$} & \multirow{3}{*}{$L I$} & \multirow{3}{*}{$L S$} & \multirow{3}{*}{$p$} & \multirow{3}{*}{$R P a$} & \multirow{3}{*}{$L i$} & \multirow{3}{*}{ Ls } \\
\hline & & \multicolumn{2}{|c|}{ Adecuado } & \multicolumn{2}{|c|}{ Escaso } & & & & & & & & \\
\hline & & $N$ & $\%$ & $N$ & $\%$ & & & & & & & & \\
\hline \multirow{3}{*}{ Ciudad } & Medellín & 438 & 32,7 & 24 & 21,1 & \multirow{3}{*}{0,00} & 3,2 & 2,0 & 5,2 & \multirow{3}{*}{0,1} & 1,4 & 0,6 & 3,2 \\
\hline & Barranquilla & 480 & 35,8 & 16 & 14 & & 5,3 & 3,0 & 9,2 & & 0,9 & 0,4 & 2,1 \\
\hline & Pasto & 422 & 31,5 & 74 & 64,9 & & 1,0 & & & & 1,0 & & \\
\hline \multirow{2}{*}{ Sexo } & Hombre & 461 & 34,4 & 41 & 36 & \multirow{2}{*}{0,74} & 1,0 & & & \multirow{2}{*}{0,2} & 1,0 & & \\
\hline & Mujer & 879 & 65,6 & 73 & 64 & & 1,1 & 0,7 & 1,6 & & 1,6 & 0,8 & 3,2 \\
\hline \multirow{2}{*}{ Estado civil } & Sin pareja & 634 & 47,3 & 88 & 77,2 & \multirow{2}{*}{0,00} & 1,0 & & & \multirow{2}{*}{0,0} & 1,0 & & \\
\hline & Con pareja & 706 & 52,7 & 26 & 22,8 & & 3,8 & 2,4 & 5,9 & & 4,1 & 1,8 & 9,4 \\
\hline \multirow{4}{*}{ Nivel educativo } & Ninguno & 125 & 9,3 & 15 & 13,2 & \multirow{4}{*}{0,01} & 1,0 & & & \multirow{4}{*}{0,5} & 1,0 & & \\
\hline & Primaria & 698 & 52,1 & 73 & 64 & & 1,1 & 0,6 & 2,1 & & 1,0 & 0,4 & 2,2 \\
\hline & Secundaria & 390 & 29,1 & 20 & 17,5 & & 2,3 & 1,2 & 4,7 & & 1,6 & 0,6 & 4,3 \\
\hline & Terciaria & 127 & 9,5 & 6 & 5,3 & & 2,5 & 1,0 & 6,8 & & 1,8 & 0,4 & 7,5 \\
\hline
\end{tabular}




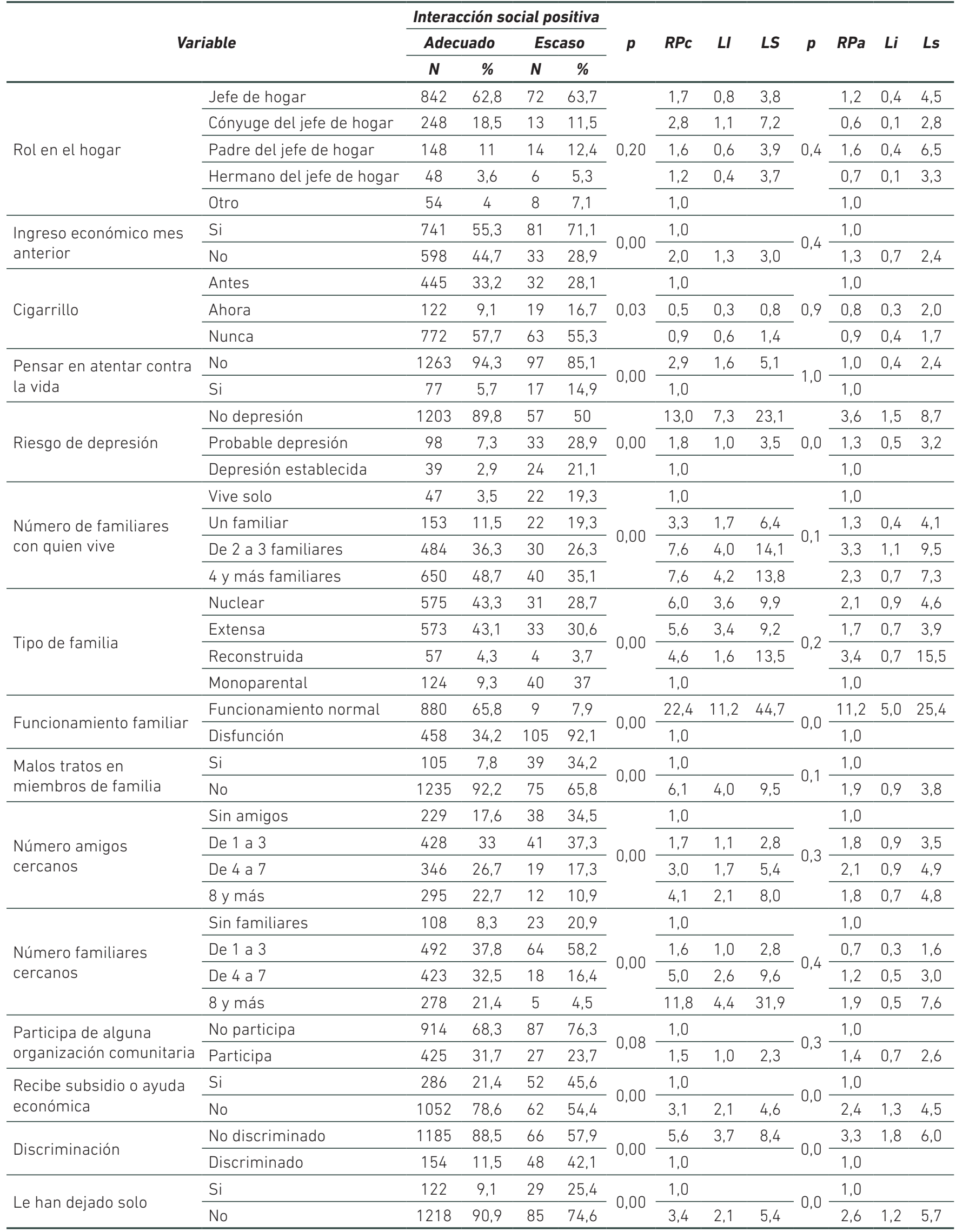




\begin{tabular}{|c|c|c|c|c|c|c|c|c|c|c|c|c|c|}
\hline & \multirow{3}{*}{ Variable } & \multicolumn{4}{|c|}{ Interacción social positiva } & \multirow{3}{*}{$p$} & \multirow{3}{*}{$R P c$} & \multirow{3}{*}{ LI } & \multirow{3}{*}{$L S$} & \multirow{3}{*}{$p$} & \multirow{3}{*}{$R P a$} & \multirow{3}{*}{$L i$} & \multirow{3}{*}{ Ls } \\
\hline & & \multicolumn{2}{|c|}{ Adecuado } & \multicolumn{2}{|c|}{ Escaso } & & & & & & & & \\
\hline & & $N$ & $\%$ & $N$ & $\%$ & & & & & & & & \\
\hline \multirow{2}{*}{ Maltrato } & No & 1234 & 92,5 & 73 & 65,2 & \multirow{2}{*}{0,00} & 6,6 & 4,3 & 10,2 & \multirow{2}{*}{0,2} & 1,7 & 0,8 & 3,5 \\
\hline & Si & 100 & 7,5 & 39 & 34,8 & & 1,0 & & & & 1,0 & & \\
\hline \multirow{2}{*}{ Tiene quien lo cuide } & Si & 223 & 16,6 & 10 & 8,8 & \multirow{2}{*}{0,03} & 2,1 & 1,1 & 4,0 & \multirow{2}{*}{0,0} & 2,7 & 1,0 & 7,2 \\
\hline & No & 1117 & 83,4 & 104 & 91,2 & & 1,0 & & & & 1,0 & & \\
\hline
\end{tabular}

*RPc: razón de prevalencias cruda; RPa: razón de prevalencias ajustada; Li: límite inferior; Ls: límite superior

\section{Apoyo afectivo}

Se identificaron diversas características de las personas mayores que se encuentran asociadas a la dimensión de apoyo afectivo. No obstante, luego de realizar un análisis ajustado para controlar factores de confusión, las variables relacionados significativamente con el adecuado apoyo afectivo fueron: tener pareja (RPa: 6,8; IC95\%: 3,3; 14,1), no presentar riesgo de depresión (RPa: 4,4; IC95\%: 1,9; 10,5), sentirse feliz (RPa: 2,9; IC95\%: 1,5; 5,3), no sentirse discriminado (RPa: 1,9; IC95\%: 1,1; 3,5), no haberlo sido dejado solo por largos periodos de tiempo (RPa: 4,5; IC95\%: 2,3; 8,8), no haber sido víctima de maltrato (RPa: 2,5 ; IC $95 \%: 1,3 ; 4,6)$ y tener quien lo cuide (RPa: 3,2; IC95\%: 1,3; 8,2) (Ver Tabla 4).

Tabla 4. Razones de prevalencia crudas y ajustadas de la dimensión apoyo afectivo de los adultos mayores de tres ciudades de Colombia, 2016

\begin{tabular}{|c|c|c|c|c|c|c|c|c|c|c|c|c|c|}
\hline & \multirow{3}{*}{ Variable } & \multicolumn{4}{|c|}{ Apoyo afectivo } & \multirow{3}{*}{$p$} & \multirow{3}{*}{$R P c$} & \multirow{3}{*}{ LI } & \multirow{3}{*}{ LS } & \multirow{3}{*}{$p$} & \multirow{3}{*}{$R P a$} & \multirow{3}{*}{$L i$} & \multirow{3}{*}{ Ls } \\
\hline & & \multicolumn{2}{|c|}{ Adecuado } & \multicolumn{2}{|c|}{ Escaso } & & & & & & & & \\
\hline & & $N$ & $\%$ & $N$ & $\%$ & & & & & & & & \\
\hline \multirow{3}{*}{ Ciudad } & Medellín & 441 & 32,5 & 21 & 21,2 & \multirow{3}{*}{0,00} & 3,0 & 1,8 & 5,0 & \multirow{3}{*}{0,0} & 3,5 & 1,7 & 6,9 \\
\hline & Barranquilla & 480 & 35,4 & 16 & 16,2 & & 4,3 & \multirow[t]{2}{*}{2,4} & 7,5 & & 1,3 & 0,6 & 2,7 \\
\hline & Pasto & 434 & 32 & 62 & 62,6 & & 1,0 & & & & 1,0 & & \\
\hline \multirow{2}{*}{ Sexo } & Hombre & 466 & 34,4 & 35 & 35,4 & \multirow{2}{*}{0,85} & 1,0 & & & \multirow{2}{*}{0,1} & 1,0 & & \\
\hline & Mujer & 889 & 65,6 & 64 & 64,6 & & 1,0 & 0,7 & 1,6 & & 1,6 & 0,9 & 3,0 \\
\hline \multirow{2}{*}{ Estado civil } & Sin pareja & 643 & 47,5 & 79 & 79,8 & \multirow{2}{*}{0,00} & 1,0 & & & \multirow{2}{*}{0,0} & 1,0 & & \\
\hline & Con pareja & 712 & 52,5 & 20 & 20,2 & & 4,4 & 2,6 & 7,2 & & 6,8 & 3,3 & 14,1 \\
\hline \multirow{4}{*}{ Nivel educativo } & Ninguno & 128 & 9,4 & 12 & 12,1 & \multirow{4}{*}{0,14} & 1,0 & & & \multirow{4}{*}{0,7} & 1,0 & & \\
\hline & Primaria & 711 & 52,5 & 61 & 61,6 & & 1,1 & 0,6 & 2,1 & & 1,0 & 0,5 & 2,4 \\
\hline & Secundaria & 390 & 28,8 & 19 & 19,2 & & 1,9 & 0,9 & 4,1 & & 1,5 & 0,6 & 3,8 \\
\hline & Terciaria & 126 & 9,3 & 7 & 7,1 & & 1,7 & 0,6 & 4,4 & & 1,0 & 0,3 & 3,1 \\
\hline \multirow{5}{*}{ Rol en el hogar } & Jefe de hogar & 847 & 62,5 & 66 & 67,3 & \multirow{5}{*}{0,14} & 1,4 & 0,6 & 3,3 & \multirow{5}{*}{0,1} & 0,5 & 0,1 & 1,9 \\
\hline & Cónyuge del jefe de hogar & 253 & 18,7 & 9 & 9,2 & & 3,0 & 1,0 & 8,8 & & 0,3 & 0,1 & 1,7 \\
\hline & Padre del jefe de hogar & 151 & 11,1 & 11 & 11,2 & & 1,5 & 0,5 & 4,2 & & 1,3 & 0,3 & 5,7 \\
\hline & Hermano del jefe de hogar & 48 & 3,5 & 6 & 6,1 & & 0,9 & 0,3 & 2,8 & & 0,3 & 0,1 & 1,5 \\
\hline & Otro & 56 & 4,1 & 6 & 6,1 & & 1,0 & & & & 1,0 & & \\
\hline \multirow{2}{*}{$\begin{array}{l}\text { Ingreso económico } \\
\text { mes anterior }\end{array}$} & $\mathrm{Si}$ & 753 & 55,6 & 68 & 68,7 & \multirow{2}{*}{0,01} & 1,0 & & 27 & & 1,0 & & \\
\hline & No & 601 & 44,4 & 31 & 31,3 & & 1,8 & 1,1 & 2,1 & 0,2 & 1,4 & 0,8 & 2,5 \\
\hline & Antes & 450 & 33,2 & 27 & 27,3 & & 1,0 & & & & 1,0 & & \\
\hline Cigarrillo & Ahora & 121 & 8,9 & 19 & 19,2 & 0,00 & 0,4 & 0,2 & 0,7 & 0,1 & 0,4 & 0,2 & 0,9 \\
\hline & Nunca & 783 & 57,8 & 53 & 53,5 & & 0,9 & 0,6 & 1,4 & & 0,9 & 0,4 & 1,6 \\
\hline
\end{tabular}




\begin{tabular}{|c|c|c|c|c|c|c|c|c|c|c|c|c|c|}
\hline & \multirow{3}{*}{ Variable } & \multicolumn{4}{|c|}{ Apoyo afectivo } & \multirow{3}{*}{$p$} & \multirow{3}{*}{$R P c$} & \multirow{3}{*}{$L I$} & \multirow{3}{*}{ LS } & \multirow{3}{*}{$p$} & \multirow{3}{*}{$R P a$} & \multirow{3}{*}{$L i$} & \multirow{3}{*}{ Ls } \\
\hline & & \multicolumn{2}{|c|}{ Adecuado } & \multicolumn{2}{|c|}{ Escaso } & & & & & & & & \\
\hline & & $N$ & $\%$ & $N$ & $\%$ & & & & & & & & \\
\hline \multirow{2}{*}{$\begin{array}{l}\text { Pensar en atentar } \\
\text { contra la vida }\end{array}$} & No & 1279 & 94,4 & 81 & 81,8 & \multirow{2}{*}{0,00} & 3,7 & \multirow[t]{2}{*}{2,1} & \multirow[t]{2}{*}{6,6} & \multirow{2}{*}{0,5} & 1,3 & \multirow[t]{2}{*}{0,6} & \multirow[t]{2}{*}{2,9} \\
\hline & Si & 76 & 5,6 & 18 & 18,2 & & 1,0 & & & & 1,0 & & \\
\hline \multirow{3}{*}{ Riesgo de depresión } & No depresión & 1213 & 89,5 & 47 & 47,5 & \multirow{3}{*}{0,00} & 12,9 & 7,1 & 23,5 & \multirow{3}{*}{0,0} & 4,4 & 1,9 & 10,5 \\
\hline & Probable depresión & 100 & 7,4 & 31 & 31,3 & & 1,6 & \multirow[t]{2}{*}{0,8} & 3,1 & & 1,0 & 0,4 & 2,4 \\
\hline & Depresión establecida & 42 & 3,1 & 21 & 21,2 & & 1,0 & & & & 1,0 & & \\
\hline \multirow{2}{*}{$\begin{array}{l}\text { Satisfacción calidad } \\
\text { de vida }\end{array}$} & Insatisfecho & 92 & 6,8 & 34 & 34,3 & \multirow{2}{*}{0,00} & 1,0 & & & \multirow{2}{*}{0,4} & 1,0 & & \\
\hline & Satisfecho & 1259 & 93,2 & 65 & 65,7 & & 7,2 & 4,5 & 11,4 & & 1,4 & 0,7 & 2,9 \\
\hline \multirow{2}{*}{ Satisfacción salud } & Insatisfecho & 184 & 13,6 & 36 & 36,4 & \multirow{2}{*}{0,00} & 1,0 & & & \multirow{2}{*}{0,7} & 1,0 & & \\
\hline & Satisfecho & 1167 & 86,4 & 63 & 63,6 & & 3,6 & 2,3 & 5,6 & & 0,9 & 0,4 & 1,7 \\
\hline \multirow{2}{*}{$\begin{array}{l}\text { Satisfacción } \\
\text { situación económica }\end{array}$} & Insatisfecho & 387 & 28,6 & 61 & 61,6 & 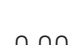 & 1,0 & & & 0 & 1,0 & & \\
\hline & Satisfecho & 964 & 71,4 & 38 & 38,4 & 0,00 & 4,0 & 2,6 & 6,1 & 0,4 & 1,3 & 0,7 & 2,3 \\
\hline Eolicidnd & Feliz & 1165 & 87,2 & 42 & 42,4 & 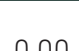 & 9,2 & 6,0 & 14,2 & مח 0 & 2,9 & 1,5 & 5,3 \\
\hline retictudu & Infeliz & 171 & 12,8 & 57 & 57,6 & 0,00 & 1,0 & & & 0,0 & 1,0 & & \\
\hline Ringrimin & No discriminado & 1192 & 88 & 59 & 59,6 & مصمص & 5,0 & 3,2 & 7,7 & مص 0 & 1,9 & 1,1 & 3,5 \\
\hline 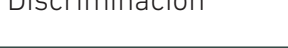 & Discriminado & 162 & 12 & 40 & 40,4 & 0,00 & 1,0 & & & 0,0 & 1,0 & & \\
\hline d & $\mathrm{Si}$ & 123 & 9,1 & 28 & 28,3 & ה مחת & 1,0 & & & O 0 & 1,0 & & \\
\hline Le nan dejado solo & No & 1232 & 90,9 & 71 & 71,7 & 0,00 & 4,0 & 2,5 & 6,4 & U,U & 4,5 & 2,3 & 8,8 \\
\hline$M=$ & No & 1247 & 92.4 & 60 & 61.9 & 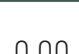 & 7,5 & 4,8 & 11,9 & مी & 2,5 & 1,3 & 4,6 \\
\hline Madtidato & Si & 102 & 7.6 & 37 & 38.1 & 0,00 & 1,0 & & & 0,0 & 1,0 & & \\
\hline & $\mathrm{Si}$ & 225 & 16,6 & 8 & 8,1 & 003 & 2,3 & 1,1 & 4,7 & 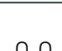 & 3,2 & 1,3 & 8,2 \\
\hline & No & 1130 & 83,4 & 91 & 91,9 & 0,03 & 1,0 & & & 0,0 & 1,0 & & \\
\hline
\end{tabular}

*RPc: razón de prevalencias cruda; RPa: razón de prevalencias ajustada; Li: límite inferior; Ls: límite superior

\section{Apoyo social}

Finalmente, con el objetivo de identificar las características de los adultos mayores tanto sociodemográficas, como familiares y de salud física y mental, que se asocian con el índice global de apoyo social, se realizaron dos análisis. En el primer análisis se identificaron múltiples variables asociadas a dicha condición. Sin embargo, con la realización del análisis ajustado para controlar factores de confusión, se identificó que las variables asociadas significativamente con un adecuado apoyo social fueron: tener pareja (RPa: 3,2; IC95\%: 1,6; 6,4), no presentar riesgo de depresión (RPa: 3,6; IC95\%: 1,3; 9,8), percibirse como una persona feliz (RPa: 2,2; IC95\%: 1,1; 4,4), convivir con 4 o más familiares (RPa: 5,7 ; IC95\%: 1,6; 20,1), tener una familia funcional (RPa: 9,4; IC95\%: 4,2; 21), ausencia de malos tratos en miembros de la familia (RPa: 2,3; IC95\%: 1,1; 4,8), tener un número de amigos cercanos superior a 4 (RPa: 3,4; IC95\%: 1,4; 8,4), no sentirse discriminado (RPa: 2,6; IC95\%: 1,3; 5,1), no haber sido dejado solo por largos periodos de tiempo (RPa: 4,0; IC95\%: 1,8; 8,9) y tener quien lo cuide (RPa: 3,4; IC95\%: 1,2; 9,1) (Ver Tabla 5). 
Tabla 5. Razones de prevalencia crudas y ajustadas apoyo social de los adultos mayores de tres ciudades de Colombia, 2016

\begin{tabular}{|c|c|c|c|c|c|c|c|c|c|c|c|c|c|}
\hline & & \multicolumn{4}{|c|}{ Apoyo social } & \multirow{3}{*}{$p$} & \multirow{3}{*}{$R P c$} & \multirow{3}{*}{$L I$} & \multirow{3}{*}{ LS } & \multirow{3}{*}{$p$} & \multirow{3}{*}{$R P a$} & \multirow{3}{*}{$L i$} & \multirow{3}{*}{ Ls } \\
\hline \multirow{2}{*}{\multicolumn{2}{|c|}{ Variable }} & \multicolumn{2}{|c|}{ Adecuado } & \multicolumn{2}{|c|}{ Escaso } & & & & & & & & \\
\hline & & $N$ & $\%$ & $N$ & $\%$ & & & & & & & & \\
\hline \multirow{3}{*}{ Ciudad } & Medellín & 436 & 32,7 & 26 & 22,2 & \multirow{3}{*}{0,00} & 2,9 & 1,8 & 4,7 & & 1,5 & 0,6 & 3,7 \\
\hline & Barranquilla & 477 & 35,7 & 17 & 14,5 & & 4,9 & \multirow[t]{2}{*}{2,9} & \multirow[t]{2}{*}{8,5} & \multirow[t]{2}{*}{0,2} & 0,6 & \multirow[t]{2}{*}{0,2} & \multirow[t]{2}{*}{1,5} \\
\hline & Pasto & 422 & 31,6 & 74 & 63,2 & & 1,0 & & & & 1,0 & & \\
\hline \multirow{2}{*}{ Sexo } & Hombre & 459 & 34,4 & 41 & 35 & 080 & 1,0 & & & 0 & 1,0 & & \\
\hline & Mujer & 876 & 65,6 & 76 & 65 & 0,01 & 1,0 & 0,7 & 1,5 & 0,0 & 1,3 & 0,6 & 2,7 \\
\hline Fstado civil & Sin pareja & 632 & 47,3 & 90 & 76,9 & 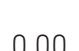 & 1,0 & & & $\cap \cap$ & 1,0 & & \\
\hline Lorand civit & Con pareja & 703 & 52,7 & 27 & 23,1 & 0,0 & 3,7 & 2,4 & 5,8 & 0,0 & 3,2 & 1,6 & 6,4 \\
\hline & Ninguno & 120 & 9 & 20 & 17,1 & & 1,0 & & & & 1,0 & & \\
\hline Nive & Primaria & 702 & 52,6 & 69 & 59 & הمח0 & 1,7 & 1,0 & 2,9 & & 1,8 & 0,8 & 4,1 \\
\hline Tivet & Secundaria & 387 & 29 & 21 & 17,9 & $U_{1}$ & 3,1 & 1,6 & 5,9 & 0,4 & 2,4 & 0,9 & 6,9 \\
\hline & Terciaria & 126 & 9,4 & 7 & 6 & & 3,0 & 1,2 & 7,4 & & 2,0 & 0,5 & 7,9 \\
\hline Ingreso económico mes & $\mathrm{Si}$ & 743 & 55,7 & 77 & 65,8 & (1) & 1,0 & & & 9 & 1,0 & & \\
\hline anterior & No & 591 & 44,3 & 40 & 34,2 & 0,04 & 1,5 & 1,0 & 2,3 & 0,7 & 1,0 & 0,5 & 2,0 \\
\hline & Antes & 441 & 33,1 & 36 & 30,8 & & 1,0 & & & & 1,0 & & \\
\hline Cigarrillo & Ahora & 122 & 9,1 & 18 & 15,4 & 0,10 & 0,6 & 0,3 & 1,0 & 0,9 & 1,2 & 0,5 & 3,0 \\
\hline & Nunca & 771 & 57,8 & 63 & 53,8 & & 1,0 & 0,7 & 1,5 & & 1,2 & 0,6 & 2,4 \\
\hline Pensar en atentar contra & No & 1259 & 94,3 & 99 & 84,6 & 0 & 3,0 & 1,7 & 5,2 & 03 & 0,6 & 0,2 & 1,5 \\
\hline la vida & $\mathrm{Si}$ & 76 & 5,7 & 18 & 15,4 & 0,0 & 1,0 & & & 0,0 & 1,0 & & \\
\hline & No depresión & 1201 & 90 & 57 & 48,7 & & 14,8 & 8,4 & 26,1 & & 3,6 & 1,3 & 9,8 \\
\hline Riesgo de depresión & Probable depresión & 97 & 7,3 & 34 & 29,1 & 0,00 & 2,0 & 1,1 & 3,8 & 0,0 & 1,1 & 0,4 & 3,2 \\
\hline & Depresión establecida & 37 & 2,8 & 26 & 22,2 & & 1,0 & & & & 1,0 & & \\
\hline Afiliado al SGSS en & $\mathrm{Si}$ & 1301 & 97,5 & 107 & 91,5 & 0,00 & 3,6 & 1,7 & 7,4 & 00 & 5,7 & 1,7 & 18,5 \\
\hline Salud & No & 34 & 2,5 & 10 & 8,5 & 0,00 & 1,0 & & & 0,0 & 1,0 & & \\
\hline Discapacidad para & No & 1152 & 86,5 & 88 & 75,2 & 000 & 2,1 & 1,3 & 3,3 & 03 & 0,7 & 0,3 & 1,5 \\
\hline escuchar & $\mathrm{Si}$ & 180 & 13,5 & 29 & 24,8 & 0,00 & 1,0 & & & 0,0 & 1,0 & & \\
\hline Diabetes o azúcar en la & No & 1142 & 85,5 & 94 & 80,3 & 0.13 & 1,4 & 0,9 & 2,3 & 6 & 1,2 & 0,6 & 2,7 \\
\hline sangre & $\mathrm{Si}$ & 193 & 14,5 & 23 & 19,7 & 0,13 & 1,0 & & & 0,0 & 1,0 & & \\
\hline & Sin riesgo & 557 & 41,9 & 30 & 25,9 & & 3,0 & 1,9 & 4,8 & & 0,7 & 0,3 & 1,6 \\
\hline Riesgo nutricional & Riesgo moderado & 375 & 28,2 & 21 & 18,1 & 0,00 & 2,9 & 1,8 & 4,9 & 0,6 & 1,1 & 0,5 & 2,4 \\
\hline & Riesgo alto & 397 & 29,9 & 65 & 56 & & 1,0 & & & & 1,0 & & \\
\hline Satisfacción calidad de & Insatisfecho & 87 & 6,5 & 39 & 33,3 & 0 & 1,0 & & & & 1,0 & & \\
\hline vida & Satisfecho & 1244 & 93,5 & 78 & 66,7 & 0,00 & 7,1 & 4,6 & 11,1 & U,L & 0,6 & 0,2 & 1,3 \\
\hline Satisfacción salud & Insatisfecho & 173 & 13 & 46 & 39,3 & 0.00 & 1,0 & & & & 1,0 & & \\
\hline satistaccion satua & Satisfecho & 1158 & 87 & 71 & 60,7 & 0,00 & 4,3 & 2,9 & 6,5 & $U, Z$ & 1,7 & 0,8 & 3,8 \\
\hline Satisfacción situación & Insatisfecho & 370 & 27,8 & 78 & 66,7 & 0.00 & 1,0 & & & , 3 & 1,0 & & \\
\hline económica & Satisfecho & 961 & 72,2 & 39 & 33,3 & 0,0 & 5,2 & 3,5 & 7,8 & 0,0 & 1,5 & 0,7 & 3,1 \\
\hline Felicidad & Feliz & 1156 & 87,8 & 50 & 42,7 & 0,00 & 9,7 & 6,5 & 14,5 & 0,0 & 2,2 & 1,1 & 4,4 \\
\hline & Infeliz & 160 & 12,2 & 67 & 57,3 & & 1,0 & & & & 1,0 & & \\
\hline & Vive solo & 44 & 3,3 & 25 & 21,4 & & 1,0 & & & & 1,0 & & \\
\hline Número de familiares & Un familiar & 152 & 11,4 & 23 & 19,7 & 000 & 3,8 & 1,9 & 7,3 & 00 & 2,4 & 0,7 & 7,9 \\
\hline con quien vive & De 2 a 3 familiares & 483 & 36,3 & 30 & 25,6 & 0,0 & 9,1 & 5,0 & 16,9 & 0,0 & 5,4 & 1,7 & 16,9 \\
\hline & 4 y más familiares & 650 & 48,9 & 39 & 33,3 & & 9,5 & 5,3 & 17,0 & & 5,7 & 1,6 & 20,1 \\
\hline
\end{tabular}




\begin{tabular}{|c|c|c|c|c|c|c|c|c|c|c|c|c|c|}
\hline \multirow{2}{*}{\multicolumn{2}{|c|}{ Variable }} & \multicolumn{4}{|c|}{ Apoyo social } & \multirow{3}{*}{$p$} & \multirow{3}{*}{$R P c$} & \multirow{3}{*}{$L I$} & \multirow{3}{*}{ LS } & \multirow{3}{*}{$p$} & \multirow{3}{*}{$R P a$} & \multirow{3}{*}{$L i$} & \multirow{3}{*}{ Ls } \\
\hline & & \multicolumn{2}{|c|}{ Adecuado } & \multicolumn{2}{|c|}{ Escaso } & & & & & & & & \\
\hline & & $N$ & $\%$ & $N$ & $\%$ & & & & & & & & \\
\hline \multirow{4}{*}{ Tipo de familia } & Nuclear & 569 & 43 & 37 & 33,3 & \multirow{4}{*}{0,00} & 4,8 & 2,9 & 7,8 & \multirow{4}{*}{0,3} & 1,0 & 0,4 & 2,2 \\
\hline & Extensa & 573 & 43,3 & 32 & 28,8 & & 5,6 & 3,4 & 9,3 & & 1,2 & 0,5 & 3,1 \\
\hline & Reconstruida & 57 & 4,3 & 3 & 2,7 & & 5,9 & 1,8 & 20,0 & & 4,7 & 0,8 & 27,3 \\
\hline & Monoparental & 125 & 9,4 & 39 & 35,1 & & 1,0 & & & & 1,0 & & \\
\hline \multirow{2}{*}{ Funcionamiento familiar } & Funcionamiento normal & 876 & 65,7 & 11 & 9,4 & \multirow{2}{*}{0,00} & 18,5 & \multirow[t]{2}{*}{9,8} & \multirow[t]{2}{*}{34,7} & \multirow{2}{*}{0,0} & 9,4 & \multirow[t]{2}{*}{4,2} & \multirow[t]{2}{*}{21,0} \\
\hline & Disfunción & 457 & 34,3 & 106 & 90,6 & & 1,0 & & & & 1,0 & & \\
\hline \multirow{2}{*}{$\begin{array}{l}\text { Malos tratos en } \\
\text { miembros de familia }\end{array}$} & $\mathrm{Si}$ & 101 & 7,6 & 43 & 36,8 & \multirow{2}{*}{0,00} & 1,0 & & & \multirow{2}{*}{0,0} & 1,0 & & \\
\hline & No & 1234 & 92,4 & 74 & 63,2 & & 7,1 & 4,6 & 10,9 & & 2,3 & 1,1 & 4,8 \\
\hline \multirow{2}{*}{$\begin{array}{l}\text { Está a cargo del cuidado } \\
\text { de otra persona }\end{array}$} & $\mathrm{Si}$ & 148 & 11,1 & 18 & 15,4 & 17 & 0,7 & 0,4 & 1,2 & & 0,5 & 0,2 & 1,0 \\
\hline & No & 1184 & 88,9 & 99 & 84,6 & 0,11 & 1,0 & & & 0,1 & 1,0 & & \\
\hline & Sin amigos & 224 & 17,3 & 43 & 38,4 & & 1,0 & & & & 1,0 & & \\
\hline Número amigos & De 1 a 3 & 425 & 32,8 & 43 & 38,4 & مחم ח & 1,9 & 1,2 & 3,0 & م م & 1,8 & 0,9 & 3,8 \\
\hline cercanos & De 4 a 7 & 348 & 26,9 & 16 & 14,3 & 0,00 & 4,2 & 2,3 & 7,6 & 0,0 & 3,4 & 1,4 & 8,4 \\
\hline & 8 y más & 297 & 23 & 10 & 8,9 & & 5,7 & 2,8 & 11,6 & & 3,4 & 1,1 & 10,4 \\
\hline & Sin familiares & 104 & 8 & 27 & 24,1 & & 1,0 & & & & 1,0 & & \\
\hline Número familiares & De 1 a 3 & 491 & 37,9 & 65 & 58 & 0 & 2,0 & 1,2 & 3,2 & & 1,2 & 0,5 & 2,7 \\
\hline cercanos & De 4 a 7 & 424 & 32,7 & 15 & 13,4 & 0,00 & 7,3 & 3,8 & 14,3 & , & 2,0 & 0,8 & 5,5 \\
\hline & 8 y más & 278 & 21,4 & 5 & 4,5 & & 14,4 & 5,4 & 38,5 & & 1,2 & 0,3 & 5,1 \\
\hline Participa de alguna & No participa & 912 & 68,4 & 87 & 74,4 & 018 & 1,0 & & & 04 & 1,0 & & \\
\hline organización comunitaria & Participa & 422 & 31,6 & 30 & 25,6 & 0,10 & 1,3 & 0,9 & 2,1 & 0,4 & 1,4 & 0,7 & 2,7 \\
\hline Recibe subsidio o ayuda & $\mathrm{Si}$ & 288 & 21,6 & 50 & 42,7 & مחمी & 1,0 & & & ח & 1,0 & & \\
\hline económica & No & 1045 & 78,4 & 67 & 57,3 & 0,00 & 2,7 & 1,8 & 4,0 & 0,0 & 2,1 & 1,0 & 4,1 \\
\hline Riscriminación & No discriminado & 1178 & 88,3 & 71 & 60,7 & 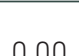 & 4,9 & 3,3 & 7,3 & חم & 2,6 & 1,3 & 5,1 \\
\hline & Discriminado & 156 & 11,7 & 46 & 39,3 & & 1,0 & & & & 1,0 & & \\
\hline 10 & Si & 118 & 8,8 & 33 & 28,2 & חी & 1,0 & & & R & 1,0 & & \\
\hline Le nan aejauo solo & No & 1217 & 91,2 & 84 & 71,8 & 0,00 & 4,1 & 2,6 & 6,3 & 0,0 & 4,0 & 1,8 & 8,9 \\
\hline Maltrato & No & 1235 & 92.9 & 70 & 60.9 & 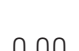 & 8,4 & 5,5 & 13,0 & 1 1 & 2,2 & 1,0 & 4,6 \\
\hline Mattrato & $\mathrm{Si}$ & 94 & 7.1 & 45 & 39.1 & 0,00 & 1,0 & & & 0,1 & 1,0 & & \\
\hline & $\mathrm{Si}$ & 223 & 16,7 & 10 & 8,5 & ? & 2,1 & 1,1 & 4,2 & 0 & 3,4 & 1,2 & 9,1 \\
\hline Trene quien lo c & No & 1112 & 83,3 & 107 & 91,5 & 0,02 & 1,0 & & & 0,0 & 1,0 & & \\
\hline
\end{tabular}

*RPc: razón de prevalencias cruda; RPa: razón de prevalencias ajustada; Li: límite inferior; Ls: límite superior

\section{Discusión}

En el presente estudio se exploraron algunos factores asociados a las dimensiones del apoyo social percibido (apoyo emocional, apoyo instrumental, interacción social positiva, apoyo afectivo, índice global de apoyo social), evaluadas mediante el cuestionario Medical Outcomes Study Social Suport Survey (Sherbourne \& Stewart, 1991), por adultos mayores de tres ciudades de Colombia. Se encontró que los adultos mayores participantes percibieron un apoyo social adecuado en sus diferentes dimensiones: 92\% emocional, 93,5\% instrumental, 92\% interacción social positiva y 93\% apoyo afectivo; porcentajes mayores de los hallados en otros estudios realizados, en Colombia en una muestra de 200 personas de 65 años y más, en el que se evidenció un adecuado apoyo afectivo del 78\%, instrumental 76\%, interacción positiva $73 \%$ y apoyo emocional 72\% (Cerquera et al., 2017); en Brasil, con una muestra de 101 personas de 60 años en el que se encontró un adecuado apoyo social en el índice global 
Pág 159

La dimensión de interacción social positiva percibida por los adultos mayores, evaluada mediante el MOS-SS, presentó una relación significativa con variables como tener pareja, no presentar síntomas que indiquen riesgo de depresión, percibir un buen funcionamiento familiar, no experimentar situaciones de discriminación, estar acompañado y tener alguien encargado de su cuidado. del 74,32\% (Brito \& Pavarini, 2012); y en México, en una muestra de 368 adultos de 60 años y más encontraron un índice de adecuado apoyo social de 78,3\% (Valdez \& Álvarez, 2018).

La percepción de un adecuado apoyo emocional de parte de los adultos mayores se relaciona con diversos factores como convivir con su pareja, sentirse feliz, no experimentar situaciones de discriminación, mantenerse acompañado, no haber recibido ingresos en el mes anterior y no presentar síntomas cognoscitivos que indiquen estar riesgo de depresión. Diversos estudios apoyan la relación positiva entre algunas de estas variables: Una investigación realizada en Argentina, con una muestra de 85 personas con edades de 65 años, resalta la importancia de la pareja en el fortalecimiento de lazos emocionales y su efecto en el retraso del deterioro cognitivo (Arias \& Polizzi, 2011). Un estudio realizado en México con personas entre 65 y 100 años de edad resalta la importancia del apoyo emocional como fuente directa de cuidado, afecto, seguridad y filiación, y como condicionante para el disfrute de la vejez, incrementando el sentimiento de felicidad (Carmona, s. f.). Por su parte, un estudio adelantado en Malaysia expone que la soledad en la vejez es un factor que propicia estadios de depresión y disminución en la calidad de vida, los cuales son mediados por la disminución en el adecuado apoyo emocional (Wan Mohd Azam et al., 2013). Además, se ha encontrado una asociación entre síntomas depresivos y bajo apoyo social (Cancino et al., 2018; Santos \& Valdés, 2018), y una oportunidad de desarrollar síntomas depresivos 4,9 veces en comparación con adultos mayores que perciben un adecuado apoyo social (Castro Lizárraga et al., 2006). Varios autores resaltan la importancia del apoyo y la construcción de redes de apoyo social desde la perspectiva de sobrellevar o afrontar, es decir, subsanar las necesidades presentadas en alguna situación desfavorable ya sea por situaciones adversas como la pérdida de empleo, catástrofes naturales, situaciones de desplazamiento, etc. (Antonucci \& Akiyama, 1987; Aranda \& Pando, 2013; Montes de Oca, 2002; Ramírez et al., 2002). Por su parte, Fuentes (2014) destaca la importancia de aceptación de la vejez, dejando de un lado los sentimientos negativos asociados a ésta, lo cual impide la percepción de apoyo de familiares y amigos; y Saideh et al., (2012) hacen hincapié en el apoyo emocional como factor predominante para mejorar la calidad de vida de los adultos mayores.

Aspectos como tener pareja, compañía constante y no ser víctima de maltrato se relacionan de manera significativa con el apoyo instrumental percibido por los adultos mayores. Otros estudios sustentan estos hallazgos: Arias y Polizzi (2011) resaltan la importancia de la pareja, la familia y los amigos, como fuente de apoyo en la vejez; la pareja como soporte en la mayoría de actividades a partir del entendimiento y la aceptación mutua, el respaldo de los hijos en lo relacionado con los aspectos prácticos y los amigos en los aspectos emocionales y cognitivos. Benites et al., (2018) exponen la relación del escaso apoyo instrumental con la presencia de maltrato en el adulto mayor, y Salinas et al., (2008) encontraron asociación entre el adecuado apoyo social y el número de vínculos que tiene una persona, de modo que en los adultos mayores que vivían solos o tenían alguna dependencia física este apoyo se percibe limitado.

La dimensión de interacción social positiva percibida por los adultos mayores, evaluada mediante el MOS-SS, presentó una relación significativa con variables como tener pareja, no presentar síntomas que indiquen riesgo de depresión, percibir un buen funcionamiento familiar, no experimentar situaciones de discriminación, estar acompañado y tener alguien encargado de su cuidado. Estos hallazgos coinciden 
El índice global del apoyo social, que incluye todas las dimensiones del apoyo percibido, en los adultos mayores se asoció con tener pareja, no tener riesgo de depresión, estar afiliado al SGSS en salud, sentirse feliz, la cantidad de familiares con los que convive, el buen funcionamiento familiar, no recibir malos tratos por parte de miembros de la familia, la cantidad de amigos que se tienen, recibir subsidio o ayuda económica, estar acompañado y contar con alguien que los cuide. con los de un estudio realizado en México, en el que se encontró que la interacción social era un factor importante para predecir el bienestar subjetivo del adulto mayor (Valdés, 2009) y otro en Colombia, que resalta la importancia de contar con una pareja permanente para alcanzar bienestar subjetivo, relacionado con la calidad de vida (Herrera et al., 2012). Otro estudio realizado en Colombia encontró una asociación entre el estado civil y la buena integración social del adulto mayor, siendo esta mayor en los solteros y los viudos, lo cual puede ser explicado por la necesidad de generación de nuevos vínculos ante la ausencia de una pareja permanente (Durán et al., 2008).

La ausencia de interacción social en los adultos mayores afecta el adecuado apoyo social, potencia la aparición de trastornos cognitivos, depresión, enfermedades crónicas múltiples, caídas y dolor de cabeza con causa incierta. Independiente de la edad y el nivel educativo, se ha encontrado que el dolor de cabeza y los síntomas depresivos en hombres sin interacción social y la aparición de caídas en mujeres fue significativamente mayor en comparación con los que si tenían interacción social ( $p$ $<0.05$ ) (Mouodi et al., 2016); y en adulos mayores, se ha evidenciado asociación entre la promoción de la interacción social y la disminución de discapacidades (Hikichi et al., 2015). Por otra parte, se han encontrado sentimientos de soledad más frecuentes en mujeres con edades entre 50 a 65 años, sin pareja, que vivían en zonas rurales, con menos interacción social (Domènech-Abella et al., 2017).

La percepción de apoyo afectivo por parte de los adultos mayores participantes en la presente investigación se asoció con tener pareja, no presentar riesgo de depresión, sentirse feliz, no sentirse discriminado, estar acompañado, no recibir maltratos y tener alguien encargado de su cuidado. En la Encuesta Nacional de Salud, Bienestar y Envejecimiento -SABE-, realizada en Colombia en el 2015, se encontró que en su mayoría los adultos mayores recibían soporte social por parte de sus hijos, resaltando que 85\% recibía apoyo emocional (Quiñones, 2017). Un estudio realizado en Argentina, señala que $88,1 \%$ de los hombres y $63,4 \%$ de las mujeres reportaron percepción de apoyo emocional por parte de su pareja (Arias \& Polizzi, 2011).

Y el índice global del apoyo social, que incluye todas las dimensiones del apoyo percibido, en los adultos mayores se asoció con tener pareja, no tener riesgo de depresión, estar afiliado al SGSS en salud, sentirse feliz, la cantidad de familiares con los que convive, el buen funcionamiento familiar, no recibir malos tratos por parte de miembros de la familia, la cantidad de amigos que se tienen, recibir subsidio o ayuda económica, estar acompañado y contar con alguien que los cuide. En este mismo sentido, otros estudios han hallado asociación entre el bienestar psicológico, la auto aceptación, propósito en la vida, relaciones positivas con otros y crecimiento personal (Vivaldi \& Barra, 2012); el apoyo social con el número de vínculos, vivir con pareja u otros familiares (Salinas et al., 2008); y con el sexo, siendo éste más favorable para las mujeres (Castillo et al., 2012); y asociación entre autoestima y satisfacción, y falta de apoyo con aislamiento social, en población institucionalizada (Antelo \& Espinosa, 2015).

Respecto a las características sociodemográficas se evidenció que ser mujer es un factor que favorece la percepción de adecuado apoyo social en el adulto mayor, así como contar con una pareja estable (Vivaldi \& Barra, 2012). Respecto a la última variable, Sherbourne y Hays (1990) señalan que estar casado proporciona un buen soporte social en aspectos como el deterioro de la salud; y Johnson (1983) destaca que la pareja proporciona un apoyo estable, completo y sin estrés. 
Pág 161

El ser humano por naturaleza es un ser sociable, por ende, en el trascurso de la vida va tejiendo vínculos que sirven de sustento, emocional, afectivo y económico, con lo cual garantiza una adecuada integración social; para el adulto mayor es de vital importancia mantener estos vínculos, que sirven para vivir de forma adecuada y con bienestar esta etapa de la vida.
Es importante resaltar la importancia del apoyo social en condiciones desfavorables. En el presente estudio se encontró una asociación entre la percepción de apoyo emocional y no haber recibido ingresos económicos en el mes anterior, la cual puede ser explicada por el hecho de que sean otros familiares y amigos los que se hagan cargo de las necesidades económicas del adulto mayor y este a su vez valore más el apoyo emocional que el económico. Un estudio sobre población adulta mayor migrante con problemas económicos en Estados Unidos resalta la importancia del apoyo emocional e instrumental, que, aparte de suplir necesidades básicas, propicia la materialización de nuevos vínculos emocionales (Yoo \& Zippay, 2012). Por otra parte, Patrick et al. (2001) resalta el modo como el apoyo emocional propicia el surgimiento de recursos subjetivos en adultos mayores con limitaciones económicas que les permite mejorar su bienestar general.

\section{Conclusiones}

El ser humano por naturaleza es un ser sociable, por ende, en el trascurso de la vida va tejiendo vínculos que sirven de sustento, emocional, afectivo y económico, con lo cual garantiza una adecuada integración social; para el adulto mayor es de vital importancia mantener estos vínculos, que sirven para vivir de forma adecuada y con bienestar esta etapa de la vida. Esto se evidenció en los resultados de la presente investigación, que destacan que una percepción de adecuado apoyo social se relaciona estrechamente con características positivas como sentimientos de felicidad y ausencia de depresión; y resaltan la importancia de establecer relaciones familiares adecuadas, que de manera directa se corresponden con la calidad en las relaciones personales de los miembros de la familia que son mayores. Además, ponen de manifiesto el papel fundamental de la pareja en el fortalecimiento de redes de apoyo en la vejez.

Estos resultados proveen información para la toma de decisiones, encaminadas a mantener aquellos factores que favorecen la adecuada percepción de apoyo social en la población de adultos mayores, como también modificar aquellos que la deterioran.

\section{Agradecimientos}

Los investigadores agradecen a las personas mayores de las tres ciudades, al igual que a las instituciones financiadoras.

\section{Conflicto de interés}

No se declara ningún conflicto

\section{Financiación}

Este proyecto de investigación fue financiado por el Departamento Administrativo de Ciencia, Tecnología e Innovación (Colciencias) de Colombia (Contrato: 122871149710), con el apoyo de la Universidad CES de Medellín, Colombia (Código Universitario: INV.082016.001). 


\section{Referencias}

Agudelo-Cifuentes, M. C., Arango, D. C., Cardona, A. S., Cardona, A. S., Rodríguez, D. M., \& Restrepo-Ochoa, D. A. (2019). Características sociales y familiares asociadas al maltrato al adulto mayor de Pasto. CES Psicología, 12(1), 32-42. doi: http:// dx.doi.org/10.21615/cesp.12.1.3

Alarcón, R. (2006). Desarrollo de una escala factorial para medir la felicidad. Interamerican Journal of Psychology, 40(1), 95-102.

Antelo, P., \& Espinosa, P. (2015). La influencia del apoyo social en usuarios institucionalizados socialmente vulnerables. Revista de Estudios e Investigación en Psicología y Educación, 2(1), 54-59. doi: https://doi.org/10.17979/reipe.2015.2.1.267

Antonucci, T. C., \& Akiyama, H. (1987). Social networks in adult life and a preliminary examination of the convoy model. Journal of Gerontology, 42(5), 519-527. doi: https://doi.org/10.1093/geronj/42.5.519

Aranda, C., \& Pando, M. (2013). Conceptualización del apoyo social y las redes de apoyo social. Revista de investigación en psicología, 16(1), 233-245.

Arias, C. (2008). El apoyo social en la vejez: Alternativas de acción frente a los desafíos del envejecimiento poblacional. Perspectivas en psicología, 5(2), 54-61.

Arias, C., \& Polizzi, L. (2011). La relación de pareja. Funciones de apoyo y sexualidad en la vejez. Revista Kairós: Gerontologia, 14, 49-71. doi: https://doi.org/10.2307/j. ctv9hvtps.10

Árraga Barrios, M. V., \& Sánchez Villarroel, M. (2011). Validez y confiabilidad de la Escala de Felicidad de Lima en adultos mayores venezolanos. Universitas Psychologica, 11(2), 381-393. doi: https://doi.org/10.11144/javeriana.upsy11-2.vcef

Arredondo, N. H. L. (2012). Validación en Colombia del cuestionario MOS de apoyo social. International Journal of Psychological Research, 5(1), 142-150.

Barrón, A. (1996). Apoyo social: Aspectos teóricos y aplicaciones. Siglo XXI de España Editores.

Beck, A. M., Ovesen, L., \& Osler, M. (1999). The 'Mini Nutritional Assessment'(MNA) and the 'Determine Your Nutritional Health'Checklist (NSI Checklist) as predictors of morbidity and mortality in an elderly Danish population. British Journal of Nutrition, 81(01), 31-36.

Bellón Saameño, J. A., Delgado Sánchez, A., Luna del Castillo, J. de D., \& Lardelli Claret, P. (1996). Validez y fiabilidad del cuestionario de función familiar Apgar-familiar. Atención Primaria, 18(6), 289-296.

Benites, M. E. V., Torres, E. F., García, M. R., Castilla, J. J. M., Arévalo, R. del P. B., Ramírez, R. V., Vásquez, A. L. V., González, Á. L., Martínez, C. D., Gaviola, Z. O., \& others. (2018). Maltrato en la vejez: Variables asociadas. Conocimiento Amazónico, 7(2), 127-141.

Brito, T. R. P. de, \& Pavarini, S. C. I. (2012). The relationship between social support and functional capacity in elderly persons with cognitive alterations. Revista Latino-Americana de Enfermagem, 20(4), 677-684. doi: https://doi.org/10.1590/ s0104-11692012000400007

Campo-Arias, A., Herazo, E., \& Oviedo, H. C. (2015). Escala de Discriminación en la Vida Cotidiana: Consistencia y estructura interna en estudiantes de medicina. Revista Médica de Risaralda, 21(2), 39-42.

Cancino, M., Rehbein-Felmer, L., Ortiz, M. S., Cancino, M., Rehbein-Felmer, L., \& Ortiz, M. S. (2018). Funcionamiento cognitivo en adultos mayores: Rol de la reserva cognitiva, apoyo social y depresión. Revista médica de Chile, 146(3), 315-322. doi: https://doi.org/10.4067/s0034-98872018000300315

Cardona A, D., Estrada R, A., \& Agudelo G, H. (2003). Envejecer nos Toca a todos. Medellín: Facultad Nacional de Salud Pública, Universidad de Antioquia. 
Cardona, D., \& Peláez, E. (2012). Envejecimiento poblacional en el siglo XXI: oportunidades, retos y preocupaciones. Revista Salud Uninorte, 28(2), 335-348.

Carmona, S. E. C. (s. f.). El apoyo y la convivencia como predictores de bienestar en la vejez. Revista Kairós Gerontologia, 15.

Castillo, Á., Albala, C., Dangour, A. D., \& Uauy, R. (2012). Factores asociados a satisfacción vital en una cohorte de adultos mayores de Santiago, Chile. Gaceta Sanitaria, 26(5), 414-420. doi: https://doi.org/10.1016/j.gaceta.2011.11.021

Castro, LM., Ramírez, ZS., Aguilar, MLV., \& Díaz de, AVM. (2006). Factores de riesgo asociados a la depresión del Adulto Mayor. Neurología, Neurocirugía y Psiquiatría, 39(4), 132-137. Recuperado de: https://www.medigraphic.com/pdfs/revneuneupsi/nnp-2006/nnp064b.pdf

Cerquera, A. M., Uribe Rodríguez, A. F., Camacho, M., Jhair, Y., \& Delgado Porras, M. P. (2017). Apoyo social percibido y dependencia funcional en el adulto mayor con dolor. Diversitas: Perspectivas en Psicología, 13(2), 215-227.

Departamento Administrativo Nacional de Estadística (DANE). (2018). Censo Nacional de Población y Vivienda 2018. Recuperado de: https://www.dane.gov.co/files/censo2018/informacion-tecnica/cnpv-2018-presentacion-3ra-entrega.pdf

Domènech-Abella, J., Lara, E., Rubio-Valera, M., Olaya, B., Moneta, M. V., Rico-Uribe, L. A., Ayuso-Mateos, J. L., Mundó, J., \& Haro, J. M. (2017). Loneliness and depression in the elderly: The role of social network. Social Psychiatry and Psychiatric Epidemiology, 52(4), 381-390. doi: https://doi.org/10.1007/s00127-017-1339-3

Durán, D. M., Valderrama, L. J. O., Uribe-Rodríguez, A. F., \& Linde, J. M. U. (2008). Social Integration and Functional Skills in Older Adults. Universitas Psychologica, 7(1), 9.

Esquivel, J. R., \& García, R. J. S. (2017). Vejez y apoyo social. Revista de educación y desarrollo, 15, 66-76.

Fuentes, C. L. C. (2014). La influencia del apoyo social en el estado emocional y las actitudes hacia la vejez y el envejecimiento en una muestra de ancianos. International Journal of Psychology, 13. doi: https://doi.org/10.1016/j.regg.2013.06.001

García, M. (2002). Beneficios del apoyo social durante el envejecimiento: Efectos protectores de la actividad y el deporte. Revista Digital, 8(51), 1-4.

Giraldo-Rodríguez, L., \& Rosas-Carrasco, O. (2013). Development and psychometric properties of the Geriatric Mistreatment Scale. Geriatrics \& Gerontology International, 13(2), 466-474.

Gómez, M., \& Sabeh, E. (2001). Calidad de vida. Evolución del concepto y su influencia en la investigación y la práctica. Instituto Universitario de Integración en la Comunidad, Facultad de Psicología, Universidad de Salamanca. doi: https://doi. org/10.15381/anales.v78i3.13766

Herrera, E. M., López, A. A., \& Pérez, B. C. (2012). Factores asociados a la calidad de vida de adultos mayores. Cartagena (Colombia). Revista Científica Salud Uninorte, 28(2). doi: https://doi.org/10.15446/rsap.v19n4.55806

Hikichi, H., Kondo, N., Kondo, K., Aida, J., Takeda, T., \& Kawachi, I. (2015). Effect of a community intervention programme promoting social interactions on functional disability prevention for older adults: Propensity score matching and instrumental variable analyses, JAGES Taketoyo study. J Epidemiol Community Health, 69(9), 905-910. doi: https://doi.org/10.1136/jech-2014-205345

Icaza, M. G., \& Albala, C. (1999). Minimental State Examinations (MMSE) del estudio de demencia en Chile: Analisis estadistico. Organizacion Panamericana de la Salud.

Johnson, C. L. (1983). Dyadic Family Relations and Social Support. The Gerontologist, 23(4), 377-383. doi: https://doi.org/10.1093/geront/23.4.377

Maslow, A. H. (1964). Synergy in the society and in the individual. Journal of Individual Psychology, 20(2), 153. 
Montes de Oca, V. (2002). Participación, organización y significado de las redes de apoyo comunitario entre hombres y mujeres adultas mayores: La experiencia de la colonia de Aragón en la Delegación Gustavo A. Madero, CD. De México. Reunión de Expertos en Redes de Apoyo Social a Personas Adultas Mayores: El Rol Del Estado, La Familia y La Comunidad. CELADE.

Mouodi, S., Bijani, A., Hosseini, S. R., \& Hajian-Tilaki, K. (2016). Gender differences in the health status of elderly living alone compared to those who are not alone: Evidence of the AHAP study, North of Iran. Caspian Journal of Internal Medicine, 7(2), 126-132.

Organización de Naciones Unidas. (2019). Perspectivas de la población mundial 2019. Recuperado de https://population.un.org/wpp/Publications/Files/WPP2019 PressRelease ES.pdf

Palomba, R. (2002). Calidad de vida: Conceptos y medidas. http://www.cepal.org/celade/agenda/2/10592/envejecimientoRP1_ppt.pdf

Patrick, J. H., Cottrell, L. E., \& Barnes, K. A. (2001). Gender, Emotional Support, and Well-Being Among the Rural Elderly. Sex Roles, 45(1), 15-29. doi: https://doi. org/10.1023/A:1013056116857

Porras-Juárez, C., Grajales-Alonso, I., Hernández-Cruz, M. C., Alonso-Castillo, M. M., \& Tenahua-Quitl, I. (2010). Percepción del adulto mayor acerca de los beneficios, barreras y apoyo social para realizar actividad física. Revista Médica del Instituto Mexicano del Seguro Social, 48(2), 127-132.

Quiñones, J. C. G. (2017). Resumen:" Sabe Colombia 2015: Estudio Nacional de Salud, Bienestar y Envejecimiento". Carta Comunitaria, 25(144), 24-35. doi: https://doi. org/10.26752/ccomunitaria.v25.n144.152

Ramírez, M. G., García, M. F. M., \& Marín, M. J. A. (2002). La elección de fuentes de apoyo social entre inmigrantes. Psicothema, 14(2), 369-374.

Ramos, J., \& Salinas, R. (2010). Vejez y apoyo social. Revista de Educación y Desarrollo, $15,69-76$.

Riaño G.D. (1991). Calidad de vida: Aproximación histórico-conceptual (30.a ed.). Boletin de Psicología, 30, 55-94.

Saideh, G., Hossein, S., \& Fatemeh, S. (2012). The Study of Relationship between Social Support and Quality of Life among Elderly People in Kerman. Jundishapur Scientific Medical Journal, 11(378), 303-315. Recuperado de: https://www.sid.ir/ en/journal/ViewPaper.aspx?id=267087

Salinas, A., Manrique, B., \& Rojo, M. T. (2008). Redes de apoyo social en la vejez: Adultos mayores beneficiarios del componente para Adultos Mayores del Programa Oportunidades. Enrique Peláez, Sociedad y adulto mayor en América Latina. Estudios de envejecimiento en la región. Río de Janeiro: Asociación Latinoamericana de Población y Fondo de Población de Naciones Unidas. Recuperado de: http://189.209.180.187/ bibliotecageriatria/acervo/pdf/ALAP 2008 FINAL 281.pdf

Santos, P. V. D. Los, \& Valdés, S. E. C. (2018). Prevalencia de depresión en hombres y mujeres mayores en México y factores de riesgo. Población y Salud en Mesoamérica, 15(2), 8.

Sheik JI, \& Yesavage JA. (1986). Geriatric Depression Scale: Recent evidence and development of a shorter version. Clin Gerontol, 5, 165-172.

Sherbourne, C. D., \& Stewart, A. L. (1991). The MOS social support survey. Social Science \& Medicine, 32(6), 705-714.

Sherbourne, Cathy D., \& Hays, R. D. (1990). Marital status, social support, and health transitions in chronic disease patients. Journal of Health and Social Behavior, 31(4), 328-343. doi: https://doi.org/10.2307/2136817

Valdés, S. E. C. (2009). El bienestar personal en el envejecimiento. Iberoforum. Revista de Ciencias Sociales de la Universidad Iberoamericana, 4(7), 48-65. 
Valdez, M. G., \& Álvarez, C. (2018). Calidad de vida y apoyo familiar en adultos mayores adscritos a una unidad de medicina familiar. Horizonte sanitario, 17(2), 9. doi: https://doi.org/10.22201/facmed.14058871p.2015.3.50727

Vivaldi, F., \& Barra, E. (2012). Bienestar Psicológico, Apoyo Social Percibido y Percepción de Salud en Adultos Mayores. Terapia Psicológica, 30(2), 23-29. doi: https://doi. org/10.4067/s0718-48082012000200002

Wan Mohd Azam, W. M. Y., Din, N. C., Ahmad, M., Ghazali, S. E., Ibrahim, N., Said, Z., ... Maniam, T. (2013). Loneliness and depression among the elderly in an agricultural settlement: Mediating effects of social support. Asia-Pacific Psychiatry, 5 , 134-139. doi: https://doi.org/10.1111/appy.12061

Yoo, J. A., \& Zippay, A. (2012). Social networks among lower income Korean elderly immigrants in the U.S. Journal of Aging Studies, 26(3), 368-376. doi: https://doi. org/10.1016/j.jaging.2012.03.005

Zavala, M., Vidal, D., Castro, M., Quiroga, P., \& Klassen, G. (2006). Funcionamiento social del adulto mayor. Ciencia y enfermería, 12(2), 53-62. doi: https://doi.org/10.4067/ $\underline{\text { s0717-95532006000200007 }}$ 